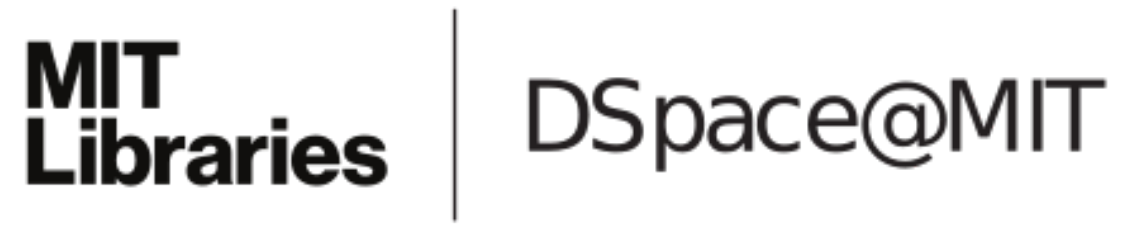

\author{
MIT Open Access Articles
}

Do Dark Pools Harm Price Discovery?

The MIT Faculty has made this article openly available. Please share how this access benefits you. Your story matters.

Citation: Zhu, H. “Do Dark Pools Harm Price Discovery?” Review of Financial Studies 27, no. 3 (March 1, 2014): 747-789.

As Published: http://dx.doi.org/10.1093/rfs/hht078

Publisher: Oxford University Press

Persistent URL: http://hdl.handle.net/1721.1/88124

Version: Author's final manuscript: final author's manuscript post peer review, without publisher's formatting or copy editing

Terms of use: Creative Commons Attribution-Noncommercial-Share Alike 


\title{
Do Dark Pools Harm Price Discovery?*
}

\author{
Haoxiang $\mathrm{Zhu}^{\dagger}$ \\ MIT Sloan School of Management
}

November 16, 2013

Forthcoming, Review of Financial Studies

\begin{abstract}
Dark pools are equity trading systems that do not publicly display orders. Dark pools offer potential price improvements but do not guarantee execution. Informed traders tend to trade in the same direction, crowd on the heavy side of the market, and face a higher execution risk in the dark pool, relative to uninformed traders. Consequently, exchanges are more attractive to informed traders, and dark pools are more attractive to uninformed traders. Under certain conditions, adding a dark pool alongside an exchange concentrates price-relevant information into the exchange and improves price discovery. Improved price discovery coincides with reduced exchange liquidity.
\end{abstract}

Keywords: dark pools, price discovery, liquidity, fragmentation, equity market structure JEL Codes: G12, G14, G18

\footnotetext{
${ }^{*}$ First version: November 2010. For helpful comments and discussions, I am very grateful to Darrell Duffie, an anonymous referee, Sal Arnuk, Jonathan Berk, John Beshears, Bradyn Breon-Drish, Robert Burns, Peter DeMarzo, Thomas George, Steven Grenadier, Frank Hatheway, Nikolaus Hautsch, David Hirshleifer (editor), Dirk Jenter, Ron Kaniel, Arthur Korteweg, Andrew Karolyi, Ilan Kremer, Charles Lee, Han Lee, Ian Martin, Jim McLoughlin, Katya Malinova, Albert Menkveld, Stefan Nagel, Maureen O’Hara, Michael Ostrovsky, Andreas Park, Francisco Pérez-González, Paul Pfleiderer, Monika Piazzesi, Mark Ready (discussant), Gideon Saar, Martin Schneider, Ken Singleton, Jeffrey Smith, Ilya Strebulaev, Ingrid Werner (discussant), Mao Ye (discussant), Ruiling Zeng, and Jeff Zwiebel, as well as seminar participants at Stanford University, the Western Finance Association annual meeting, the NBER Market Design Working Group meeting, Chicago Booth, Princeton University, University of Illinois at Urbana-Champaign, MIT Sloan, NYU Stern, Wharton, UT Austin McCombs, Berkeley Haas, UCLA Anderson, Northwestern Kellogg, the SFS Finance Cavalcade, the NY Fed Money and Payments Workshop, the Federal Reserve Board, Cornell University, Stockholm School of Economics, Humboldt University Berlin, Boston University, University of Illinois at Chicago, and Vanderbilt University. Working paper URL: http://ssrn.com/abstract=1712173.

${ }^{\dagger}$ Send correspondence to Haoxiang Zhu, MIT Sloan School of Management, 100 Main Street E62-623, Cambridge, MA 02142, USA; telephone: +1(617)-253-2478. E-mail: zhuh@mit.edu.
} 
Dark pools are equity trading systems that do not publicly display orders. Some dark pools passively match buyers and sellers at exchange prices, such as the midpoint of the exchange bid and offer. Other dark pools execute orders by their price and time priority. According to the Securities and Exchange Commission (SEC 2010), as of September 2009, thirty-two dark pools in the United States accounted for $7.9 \%$ of total equity trading volume. As of mid-2011, industry estimates from the Tabb Group, a consultancy, and Rosenblatt Securities, a broker, attribute about $12 \%$ of U.S. equity trading volume to dark pools. The market shares of dark pools in Europe, Canada, and Asia are smaller but are quickly growing (see Section 1 and International Organization of Securities Commissions 2010).

Dark pools have raised regulatory concerns in that they may harm price discovery. The European Commission (2010), for example, remarks that "[a]n increased use of dark pools ... raise $[\mathrm{s}]$ regulatory concerns as it may ultimately affect the quality of the price discovery mechanism on the 'lit' markets." The International Organization of Securities Commissions (2011) similarly worries that "the development of dark pools and use of dark orders could inhibit price discovery if orders that otherwise might have been publicly displayed become dark." According to a survey conducted by the CFA Institute (2009), 71\% of respondents believe that the operations of dark pools are "somewhat" or "very" problematic for price discovery. The Securities and Exchange Commission (2010), too, considers "the effect of undisplayed liquidity on public price discovery" an important regulatory question. Speaking of nondisplayed liquidity, SEC Commissioner Elisse Walter commented that " $\mathrm{t}]$ here could be some truth to the criticism that every share that is crossed in the dark is a share that doesn't assist the market in determining an accurate price." ${ }^{1}$

In this paper I investigate the impact of dark pools on price discovery. Contrary to misgivings expressed by some regulators and market participants, I find that under natural conditions adding a dark pool improves price discovery.

My inquiry into dark pools builds on a model of strategic venue selection by informed and liquidity traders. Informed traders hope to profit from proprietary information regarding the value of the traded asset, whereas liquidity traders wish to meet their idiosyncratic liquidity needs. Both types of traders optimally choose between an exchange and a dark pool. The exchange displays a bid price and an ask price and executes all submitted orders at the bid or the ask. The dark pool uses exchange prices and match orders at the midpoint of the exchange bid and ask. Unlike the exchange, the dark pool has no market makers through which to absorb excess order flow and

\footnotetext{
1 "Speech by SEC Commissioner: Opening Remarks Regarding Dark Pools," October 21, 2009.
} 
thus cannot guarantee execution. Sending an order to the dark pool therefore involves a trade-off between potential price improvement and the risk of no execution.

Execution risk in the dark pool drives my results. Because matching in the dark pool depends on the availability of counterparties, some orders on the "heavier" side of the market - the side with more orders - will fail to be executed. These unexecuted orders may suffer costly delays. Because informed orders are positively correlated with the value of the asset and therefore with each other, informed orders are more likely to cluster on the heavy side of the market and suffer lower execution probabilities in the dark pool. By contrast, liquidity orders are less correlated with each other and less likely to cluster on the heavy side of the market; thus, liquidity orders have higher execution probabilities in the dark pool. This difference in execution risk pushes relatively more informed traders into the exchange and relatively more uninformed traders into the dark pool. Under natural conditions, this self-selection lowers the noisiness of demand and supply on the exchange and improves price discovery. Moreover, the reporting of trading volume in dark pools further improves price discovery above and beyond the self-selection mechanism.

This price-discovery effect of dark pools complements their "size discovery" function, by which large institutional orders are executed without being revealed to the broad market. $^{2}$ This size-discovery benefit of dark trading has been widely acknowledged by market participants and regulators, and today only a handful of dark pools execute large orders (Securities and Exchange Commission 2010; Ready 2012)

The potential improvement in price discovery by dark pools should be balanced by a few other considerations. First, although dark pools tend to improve price discovery on average, they may occasionally exacerbate misleading inferences of the asset value, especially in the unlikely event that liquidity traders push the net order flow far opposite of the informed traders. Second, better price discovery need not coincide with higher liquidity. Indeed, more informative orders tend to worsen adverse selection on the exchange, leading to wider spreads and higher price impacts. Third, the longer the horizon of private information, the less effective the dark pool is in improving price discovery. Fourth, for analytical tractability I have abstracted from some of the trading practices that are applied in dark pools, such as "pinging," 3 order routing, ${ }^{4}$

\footnotetext{
${ }^{2}$ Investors who reveal their trading intentions to the market may be subject to "predatory trading," as modeled by Brunnermeier and Pedersen (2005) and Carlin, Lobo, and Viswanathan (2007).

3 "Pinging" orders are marketable orders that seek to interact with displayed or nondisplayed liquidity. Pinging is sometimes used to learn about the presence of large hidden orders.

${ }^{4}$ Order routing means sending orders from venue to venue, typically by algorithms. For example, if a dark pool cannot execute an order because there is no counterparty, the dark pool can route the order to another dark pool, which may further route the order into the market.
} 
"indication of interest" (IOI), ${ }^{5}$ and fair access. ${ }^{6}$ These practices may well contribute to controversies surrounding dark pools. Moreover, insufficient or inaccurate disclosure by dark pools of their operating mechanisms may prevent investors from making informed decisions of order execution. ${ }^{7}$

I do not claim that dark pools improve welfare. The welfare implications of dark pools depend not only on the trade-off between price discovery and liquidity, but also on how price discovery and liquidity affect production decisions, asset allocation, and capital formation. ${ }^{8}$ Although a thorough model nesting all these elements is outside the scope of this paper, the results of this paper can naturally serve as a building block for future research on the welfare implications of dark pools.

My theoretical results yield a number of empirical implications. For example, the model predicts that a higher volatility increases informed participation in dark pools, but can reduce dark-pool market share; and that the addition of a dark pool tends to increase order informativeness, spreads, and price impacts on exchanges. These predictions are broadly consistent with recent empirical evidence, as I discuss in more

\footnotetext{
${ }^{5} \mathrm{An}$ IOI is an electronic message that contains selected information (such as the ticker) about an order and is sent by a trading venue (such as a dark pool or a broker) to a selected group of market participants to facilitate a match. The Securities and Exchange Commission (2009) proposes to treat actionable IOIs - IOIs containing the symbol, size, side, and price of an order - as quotes, which must be disseminated to the broad market immediately. Buti, Rindi, and Werner (2011a) model a market in which selected traders are informed of the state of the dark pool.

${ }^{6} \mathrm{~A}$ trading venue has fair access if it allows all broker-dealers to participate. Fair access involves the subtle trade-off between excluding certain market participants and leaking information of institutional orders. In a speech on April 19, 2010, SEC Deputy Director James Brigagliano observed that "some dark pools attempt to protect institutional trading interest by raising access barrier to the sell-side or certain hedge funds." Results from Boni, Brown, and Leach (2012) indicate that the exclusion of short-term traders in a dark pool improves the execution quality of institutional orders. Foster, Gervais, and Ramaswamy (2007) theoretically illustrate that setting a volume threshold in the dark pool - that is, the dark pool executes orders only if trading interests on both sides of the market reach that threshold - can sometimes prevent impatient traders or informed traders from participating in the dark pool.

${ }^{7}$ For example, in October 2011, SEC finds that Pipeline, a dark-pool operator that claimed to only allow buyside firms to participate, had filled the majority of customer orders through its own trading affiliate (see www.sec.gov/news/press/2011/2011-220.htm). In October 2012, the SEC charged eBX for failing to protect confidential information of its subscribers (see www.sec.gov/news/press/2012/2012-204.htm). In May 2013, the Financial Industry Regulatory Authority (FINRA) requested information from a number of dark pools regarding their operation mechanisms and practice of handling customer orders (see www.finra.org/Industry/Regulation/Guidance/TargetedExaminationLetters/P268091). In a survey of 64 institutional investors, Bennett, Colon, Feng, and Litwin (2010) find that on many occasions dark pools do not disclose sufficient information of their operations to the customers. The International Organization of Securities Commissions (2010) also observes that "[l]ack of information about the operations of dark pools and dark orders may result in market participants making uninformed decisions regarding whether or how to trade within a dark pool or using a dark order."

${ }^{8}$ For example, O'Hara (2003) argues that price discovery and liquidity have important implications for asset prices. Bond, Edmans, and Goldstein (2012) survey the extensive literature on the effect of financial market for the real economy; they point out that information revealed in financial markets helps managers make real decisions, and this feedback effect can potentially explain many market phenomena that otherwise may seem puzzling.
} 
detail in Section 4.

To the best of my knowledge, this paper is the first to show that adding a dark pool can improve price discovery. My finding stands in contrast to that of Ye (2011), who studies the venue choice of a large informed trader in the Kyle (1985) framework and concludes that the addition of a dark pool harms price discovery on the exchange. Ye (2011), however, assumes that only the informed trader can freely select trading venues, whereas I allow both informed and liquidity traders to select venues.

A couple of existing models of dark pools focus on questions other than price discovery. Degryse, Van Achter, and Wuyts (2009) build a dynamic model of a dark pool and analyze how various transparency requirements for dark-pool orders affect traders' behavior and welfare. Buti, Rindi, and Werner (2011a) model the competition between an open limit order book and a dark pool, and focus on the interaction between darkpool trading and characteristics of the limit order book, such as quote depths. Because these two papers do not model asymmetric information regarding the asset value, their results and mine are complementary.

Finally, my results complement those of Hendershott and Mendelson (2000) (HM), who model the coexistence of a dealers' market (similar to the exchange in this paper) and a "crossing network" (similar to the dark pool in this paper). HM have the important insight that traders in dark pools impose positive ("liquidity-begets-liquidity") and negative ("crowding out") externalities on each other. My focus on price discovery complements HM's focus on liquidity externality.

My model differs from HM's in the behavior of exchange prices and informed traders. Specifically, informed traders in HM's model use a corner strategy: either they all go to the exchange immediately, or they all try the dark pool before the exchange. This behavior is a result of HM's assumption that, if informed traders fail to execute their orders in the dark pool, dealers are still willing to execute the leftover orders at the original quotes. ${ }^{9}$ By contrast, in my model, informed traders who fail to cross in the dark pool will face a new, potentially worse price on the exchange. This distinction regarding price risk is important for price discovery and generates evidently different empirical predictions. First, HM predicts that depending on information horizon, all

\footnotetext{
${ }^{9}$ More specifically, HM model the cost of dark-pool execution as a fraction $1-\delta$ of a trader's gain from trade. In their model, either all informed traders have $\delta=0$ (with the probability $\gamma$ ), or all informed traders have $\delta=1$ (with probability $1-\gamma$ ). In the former case (i.e., short-lived information), all informed traders immediately go to the exchange by assumption; in the latter case (i.e., long-lived information), all informed traders go to the dark pool because exchange prices do not change later. Put differently, by going to the dark pool, informed traders in HM lose either none of their information advantage or all of their information advantage. In addition, the exchange spread in HM is set once and does not change over time. In my model, informed traders choose their trading venue in a more endogenous fashion, and exchanges prices change over time.
} 
informed traders only use one venue at a time; my model predicts that informed traders (partially) use both venues regardless of information horizon. Second, in HM, informed traders' strategies are invariant to market conditions, such as volatility; in my model informed traders' strategies endogenously respond to volatility and other market conditions. Third, HM predict that if private information expires sooner, then informed traders use the exchange more, and the exchange spread becomes narrower; I show that the opposite can happen in my model. Finally, in HM the exchange spreads do not change after a dark pool crosses, whereas in my model they do. These distinct predictions can be tested empirically.

\section{An Overview of Dark Pools}

This section provides an overview of dark pools. I discuss why dark pools exist, how they operate, and what distinguishes them from each other. For concreteness, I tailor this discussion for the market structure and regulatory framework in the United States. Dark pools in Europe, Canada, and Asia operate similarly.

Before 2005, dark pools had low market share. Early dark pools were primarily used by institutions to trade large blocks of shares without revealing their intentions to the broad market, in order to avoid being front-run. A watershed event for the U.S. equity market was the adoption in 2005 and full implementation in 2007 of Regulation National Market System, or "Reg NMS" (Securities and Exchange Commission 2005), which abolished rules that had protected the manual quotation systems of incumbent exchanges. In doing so, Reg NMS encouraged newer and faster electronic trading centers to compete with the incumbents. Since Reg NMS came into effect, a wide variety of trading centers have been established. As of September 2009, the United States had about 10 exchanges, 5 electronic communication networks (ECNs), 32 dark pools, and over 200 broker-dealers (Securities and Exchange Commission 2010). Exchanges and ECNs are referred to as transparent, or "lit," venues; dark pools and broker-dealer internalization are considered opaque, or "dark," venues. In Europe, the adoption in 2007 of the Markets in Financial Instruments Directive (MiFID) similarly led to increased competition and a fast expansion of equity trading centers. ${ }^{10}$

Figure 1 shows the consolidated volume of U.S. equity markets from July 2008 to June 2011, as well as the market share of dark pools during the same periods, estimated

\footnotetext{
${ }^{10}$ For example, according to the CFA Institute (2009), European equity markets had 92 regulated markets (exchanges), 129 "multilateral trading facilities" (MTFs), and 13 "systematic internalizers" as of September 2010. For more discussion of MiFID and European equity market structure, see the European Commission (2010).
} 
Figure 1: U.S. equity trading volume and the market share of dark pools The left axis plots the daily consolidated equity trading volume in the United States, estimated by Tabb Group. The right axis plots the market shares of dark pools as a percentage of the total consolidated volume, estimated by Tabb Group and Rosenblatt Securities.

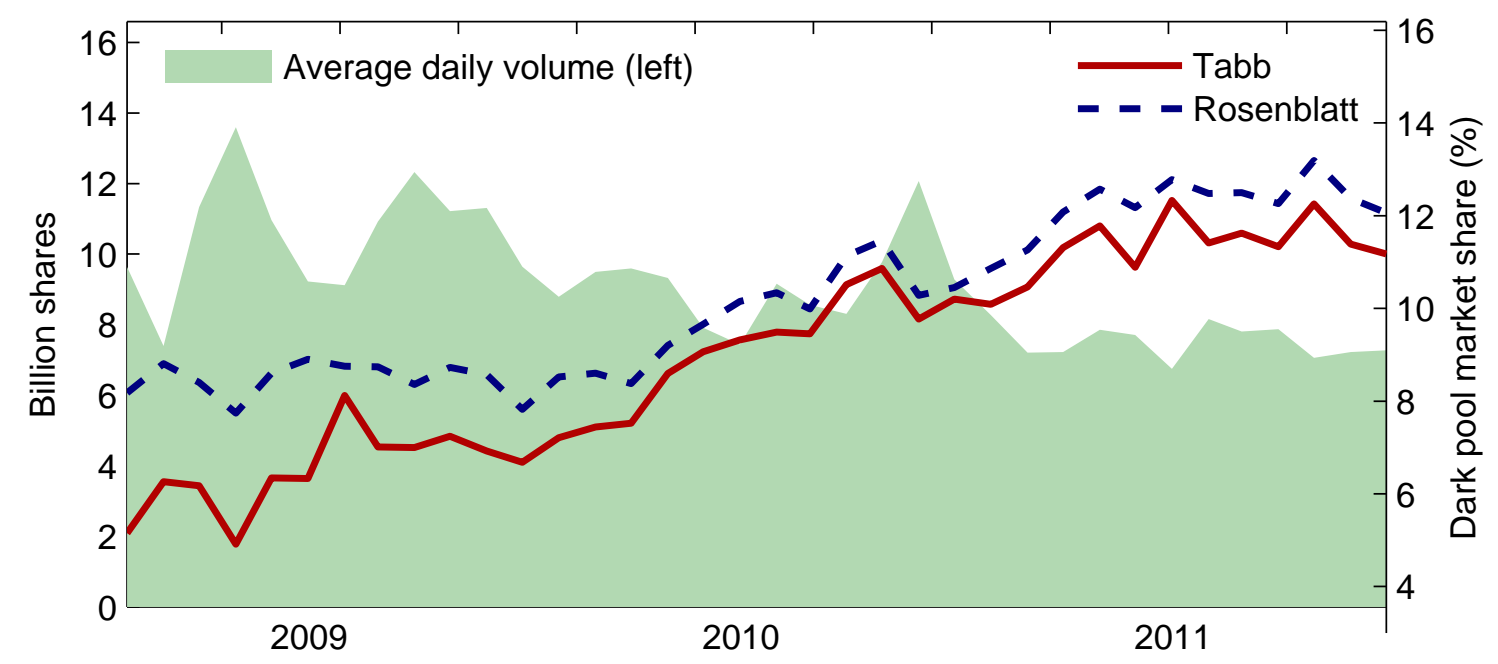

by Tabb Group and Rosenblatt Securities. According to their data, the market share of dark pools roughly doubled from about $6.5 \%$ in 2008 to about $12 \%$ in 2011 , whereas consolidated equity volume dropped persistently from about ten billion shares per day in 2008 to about seven billion shares per day in 2011. A notable exception to the decline in consolidated volume occurred around the "Flash Crash" of May 2010.

Dark pools have gained market share for reasons that go beyond recent regulations designed to encourage competition. Certain investors, such as institutions, simply need nondisplayed venues to trade large blocks of shares without alarming the broad market. This need has increased in recent years as the order sizes and depths on exchanges have declined dramatically (Chordia, Roll, and Subrahmanyam 2011). Further, dark pools attract investors by offering potential price improvements relative to the best prevailing bid and offer on exchanges. Finally, broker-dealers handling customer orders have strong incentives to set up their own dark pools, where they can better match customer orders internally and therefore save trading fees that would otherwise be paid to exchanges and other trading centers.

An important commonality among dark pools is that they derive execution prices from lit venues. For example, a typical, classical form of dark pools matches customer orders at prices derived from lit venue, such as the midpoint of the national best bid and offer $(\mathrm{NBBO})$ or the volume-weighted average price (VWAP). These dark pools 
are typically operated by "agency brokers" (broker-dealers without proprietary order flows) and exchanges. Examples include ITG Posit and Liquidnet (see also Ready 2012 for a discussion of these two dark pools), as well as midpoint dark order types offered by NASDAQ, BATS, and DirectEdge. By taking lit-venue prices as given, these dark pools do not provide direct price discovery. The model of this paper is based on this trading mechanism. ${ }^{11}$

In addition to those "midpoint" dark pools, a second group of dark pools can provide limited price discovery. Mostly operated by large broker-dealers, these dark pools are essentially continuous nondisplayed limit order books, where the execution prices are bounded between the National Best Bid and Offer. Yet a third group of dark pools operate as electronic market makers that accept or reject customer orders at high speed. To preserve space, I delegate the discussion of these dark pools, as well as additional institutional details of dark liquidity, to the Online Appendix of this paper. Overviews of dark pools and nondisplayed liquidity can also be found in Johnson (2010), Butler (2007), Carrie (2008), Securities and Exchange Commission (2010), European Commission (2010), CSA/IIROC (2009), and International Organization of Securities Commissions (2011).

\section{Modeling the Exchange and the Dark Pool}

This section presents a two-period model of trading-venue selection. As mentioned in Section 1, the dark pool modeled in this paper passively matches orders at the midpoint of the exchange's bid and ask. A glossary of key model variables can be found in Appendix A. All proofs are provided in Appendix B.

\subsection{Markets and traders}

There are two trading periods, denoted by $t=1,2$. At the end of period 2 , an asset pays an uncertain dividend $v$ that is equally likely to be $+\sigma$ or $-\sigma$. Thus, $\sigma>0$ is the volatility of the asset value. The asset value $v$ is publicly revealed at the beginning of period 2. (Longer-lived information is considered in Section 3.2.) For example, this revelation of private information may represent an earnings announcement.

Two trading venues operate in parallel: a lit exchange and a dark pool. The exchange is open in periods 1 and 2 . On the exchange, a risk-neutral market maker sets

\footnotetext{
${ }^{11}$ This type of dark pool is also the prototype of existing models, including Hendershott and Mendelson (2000), Degryse, Van Achter, and Wuyts (2009), Ye (2011), and Buti, Rindi, and Werner (2011a).
} 
competitive bid and ask prices. Market orders sent to the exchange arrive simultaneously. Exchange buy orders are executed at the ask; exchange sell orders are executed at the bid. After period-1 orders are executed, the market maker announces the volume $V_{b}$ of exchange buy orders and the volume $V_{s}$ of exchange sell orders. The market maker also announces the exchange closing price $P_{1}$, which is the expected asset value, conditional on $V_{b}$ and $V_{s}$. (In Section 3.1, the market maker also uses the dark pool trading volume to calculate $P_{1}$.) The closing price $P_{1}$ is also the price at which the market maker is willing to execute a marginal order at the end of period 1. A key objective of this section is to analyze price discovery, that is, the informativeness of these announcements, in particular $P_{1}$, for the fundamental value $v$ of the asset.

The dark pool executes (or "crosses") orders in period 1 and is closed in period 2. Closing the dark pool in period 2 is without loss of generality because once the dividend $v$ is announced in period 2, exchange trading is costless. An order submitted to the dark pool is not observable to anyone but the order submitter. The execution price of dark pool trades is the midpoint of the exchange bid and ask - also known simply as the "midpoint" or "midmarket" price. In the dark pool, orders on the "heavier side" - the buyers' side if buy orders exceed sell orders, and the sellers' side if sell orders exceed buy orders - are randomly selected for matching with those on the "lighter" side. For example, if the dark pool receives $Q_{B}$ buy orders and $Q_{S}<Q_{B}$ sell orders, all of the same size, then $Q_{S}$ of the $Q_{B}$ buy orders are randomly selected, equally likely, to be executed against the $Q_{S}$ sell orders at the midmarket price. Unmatched orders are returned to the order submitter at the end of period 1. As described in Section 1, this midpoint execution method is common in dark pools operated by agency brokers and exchanges.

For-profit traders and liquidity traders, all risk-neutral, arrive at the beginning of period 1. There is an infinite set of infinitesimal traders of each type. For-profit traders have a mass of $\bar{\mu}>0$ and can potentially trade one unit of the asset per capita. (Trading one unit per capita simplifies the exposition, and the equilibrium applies to any finite order size per capita, with minor adjustment; see Lemma 1 below.) Forprofit traders can acquire, at a cost, perfect information about $v$, and thus become informed traders. These information-acquisition costs are distributed across for-profit traders, with a differentiable cumulative distribution function $F:[0, \infty) \rightarrow[0,1]$. After observing $v$, informed traders submit buy orders (in either venue) if $v=+\sigma$ and submit sell orders if $v=-\sigma$. For-profit traders who do not acquire the information do not trade. I let $\mu_{I}$ be the mass of informed traders; their signed trading interest is therefore $Y=\operatorname{sign}(v) \cdot \mu_{I}$. 
Liquidity buyers and liquidity sellers arrive at the market separately (not as netted). The mass $Z^{+}$of liquidity buy orders and the mass $Z^{-}$of liquidity sell orders are nonnegative, independent, and identically distributed on $[0, \infty)$ with positive and finite density functions, and are infinitely divisible. Infinite divisibility means that, for each integer $n$, the total liquidity buy orders $Z^{+}$can be viewed as the aggregate demand by $n$ liquidity buyers, whose order sizes are independently and identically distributed random variables. A similar construction applies for the total liquidity sell orders $Z^{-}$. Thus, we can interpret a market with infinitely many liquidity traders as the "limiting case" of a market with $n$ liquidity buyers and $n$ liquidity sellers as $n \rightarrow \infty$. $^{12}$ In particular, because, in the limit, each liquidity trader's order size has zero mean and zero variance, the conditional joint distribution of $Z^{+}$and $Z^{-}$, given this liquidity trader's order size, is the same as the unconditional joint distribution of $Z^{+}$and $Z^{-}{ }^{13}$ I denote by $0.5 \mu_{z}$ the mean of $Z^{+}$(and $Z^{-}$) and by $0.5 \sigma_{z}^{2}$ the variance of $Z^{+}$(and $Z^{-}$).

Liquidity traders incur delay costs if they do not complete their orders in period 1. Specifically, for each liquidity trader $i$, the delay cost per unit of asset per capita is:

$$
c_{i}=\gamma_{i} \sigma
$$

where the multiplicative constants $\left\{\gamma_{i}\right\}$ represent the "types" of liquidity traders and have a twice-differentiable cumulative distribution function $G:[0, \Gamma) \rightarrow[0,1]$, for some $\Gamma \in(1, \infty]$. A linear delay cost simplifies the analysis and does not change my main result regarding price discovery. (An equilibrium characterization under constant

\footnotetext{
${ }^{12}$ More specifically, for each integer $n, Z^{+}$can be constructed as the sum of $n$ independently and identically distributed random variables $\left\{Z_{i n}^{+}\right\}$. That is,

$$
Z^{+} \sim \sum_{i=1}^{n} Z_{i n}^{+}
$$

Note that the distribution of $Z_{i n}^{+}$depends on $n$. I assume that the variance of $Z_{i n}^{+}$is finite. Similarly, there exist $n$ i.i.d. random variables $Z_{i n}^{-}$such that

$$
Z^{-} \sim \sum_{i=1}^{n} Z_{i n}^{-}
$$

In this setting, $\left\{Z_{i n}^{+}\right\}$and $\left\{Z_{i n}^{-}\right\}$can be viewed as the order sizes of $n$ liquidity buyers and $n$ liquidity sellers. As $n \rightarrow \infty$, the mean and variance of $Z_{i n}^{+}$and $Z_{i n}^{-}$converge to zero, and liquidity buyers and sellers become infinitesimal.

${ }^{13}$ We denote the probability distribution of $Z^{+}$by $\Phi$ and show that, for each $i$, the conditional distribution of $\sum_{j=1}^{n} Z_{j n}^{+}$, given $Z_{i n}^{+}$, converges to the prior distribution of $Z^{+}$as $n \rightarrow \infty$. That is, for all $z>0, \Phi\left(z \mid Z_{i n}^{+}\right) \rightarrow \Phi(z)$ as $n \rightarrow \infty$. By the independence of $Z_{i n}^{+}$and $\left\{Z_{j n}^{+}\right\}_{j \neq i}$, this amounts to showing that $Z_{i n}^{+}$converges to zero in distribution. Indeed, for any $z>0$, using Markov's inequality and the fact that $\mathbb{E}\left(Z_{i n}^{+}\right)$converges to zero as $n \rightarrow \infty$, we have

$$
\mathbb{P}\left(Z_{\text {in }}^{+}<z\right)=1-\mathbb{P}\left(Z_{\text {in }}^{+} \geq z\right) \geq 1-\frac{\mathbb{E}\left(Z_{\text {in }}^{+}\right)}{z} \rightarrow 1 \text {, as } n \rightarrow \infty .
$$

Similarly, the conditional distribution of $Z^{-}$, given $Z_{i n}^{+}$, converges in $n$ to the prior distribution of $Z^{-}$. The proof for a liquidity seller's inference is symmetric.
} 
Figure 2: Time line of the two-period model.

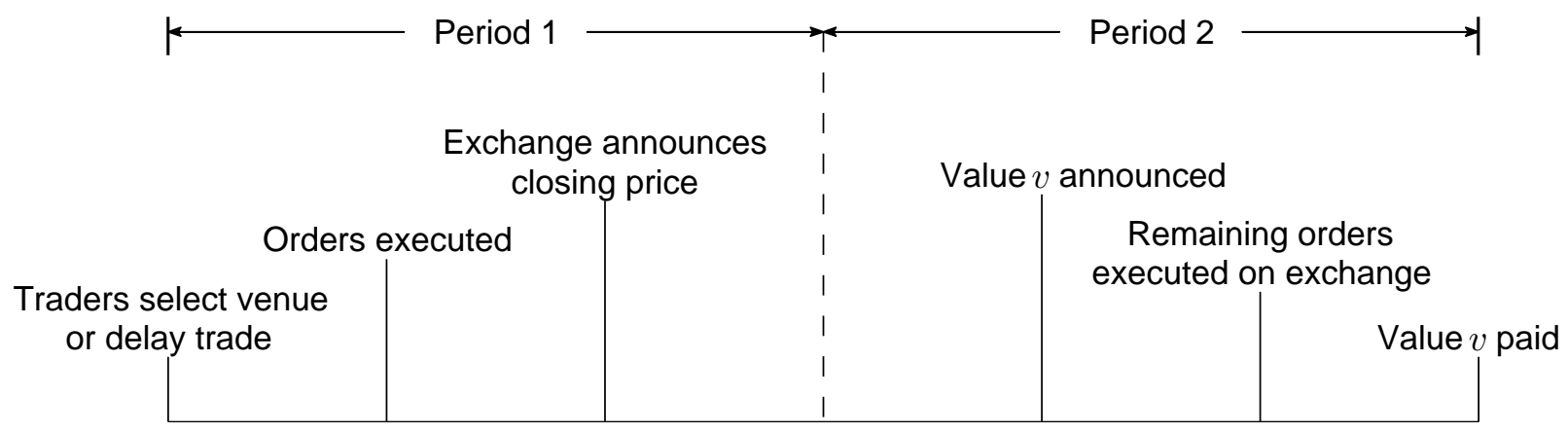

delay costs is available in an Online Appendix.) This linear delay cost can come from margin constraint or unmodeled risk aversion. In Brunnermeier and Pedersen (2009), for example, the required margin on a trade is linearly increasing in the volatility of the asset. The delay costs of informed traders, by contrast, stem from the loss of profitable trading opportunities after $v$ is revealed in period 2. (Clearly, if informed traders were subject to additional delay costs, they would be even more likely to trade on the exchange, strengthening my results.)

Finally, random variables $v, Z^{+}, Z^{-}$, and the costs of information-acquisition and delay are all independent, and their probability distributions are common knowledge. Realizations of $Y, Z^{+}$and $Z^{-}$are unobservable, with the exception that informed traders observe $v$, and hence know $Y$. Informed and liquidity traders cannot post limit orders on the exchange; they can trade only with the exchange market maker or by sending orders to the dark pool. Combining limit order books with dark pools is analytically hard and is left for future research. ${ }^{14,15}$ Further, both venues do not charge trading fees; this zero-fee assumption is made for tractability. Analyzing endogenous trading fees, which can potentially interact with the self-selection mechanism, is left for future research.

Figure 2 illustrates the sequence of actions in the two-period model.

\footnotetext{
${ }^{14}$ Limit order books are hard to solve under asymmetric information. Most models of limit order books have pure private values and do not have asymmetric information regarding the asset value. Papers taking this approach include Parlour (1998), Foucault (1999), Foucault, Kadan, and Kandel (2005), Goettler, Parlour, and Rajan (2005), Rosu (2009), and Buti, Rindi, Wen, and Werner (2011), among others. Chakravarty and Holden (1995), Kaniel and Liu (2006), and Goettler, Parlour, and Rajan (2009) allow informed traders, but incorporating a dark pool into their models is far from trivial. For a literature review of limit order books, see Parlour and Seppi (2008).

${ }^{15}$ Although limit order books also feature the trade-off between price improve and execution risk, limit order books are typically transparent. To the extent that informed traders post limit orders, depths on limit order books may reveal information. Dark pools do not reveal information through depth. Therefore, the trade-off faced by an informed trader in a dark pool is different from the trade-off in an open limit order book.
} 


\subsection{Equilibrium}

An equilibrium consists of the quoting strategy of the exchange market maker, the market participation strategies of for-profit traders, and the trading strategies of informed and liquidity traders. In equilibrium, the competitive market maker breaks even in expectation, and all traders maximize their expected net profits.

Specifically, I let $\alpha_{e}$ and $\alpha_{d}$ be candidates for the equilibrium fractions of liquidity traders who, in period 1 , send orders to the exchange and to the dark pool, respectively. The remainder, $\alpha_{0}=1-\alpha_{e}-\alpha_{d}$, choose not to submit orders in period 1 and delay trade until period 2. I let $\beta$ be the period 1 fraction of informed traders who send orders to the dark pool. The remaining fraction $1-\beta$ of informed traders trade on the exchange. (Obviously, informed traders never delay their trades as they will have lost their informational advantage by period 2.) Once the asset value $v$ is revealed in period 2, all traders who have not traded in period 1-including those who deferred trading and those who failed to execute their orders in the dark pool-trade with the market maker at the unique period 2 equilibrium price of $v$.

I first derive the equilibrium exchange bid and ask, assuming equilibrium participation fractions $\left(\beta, \alpha_{d}, \alpha_{e}\right)$. Because of symmetry and the fact that the unconditional mean of $v$ is zero, the midpoint of the market maker's bid and ask is zero. Therefore, the exchange ask is some $S>0$, and the exchange bid is $-S$, where $S$ is the exchange's effective spread, the absolute difference between the exchange transaction price and the midpoint. For simplicity, I refer to $S$ as the "exchange spread." Given the participation fractions $\left(\beta, \alpha_{d}, \alpha_{e}\right)$, the mass of informed traders on the exchange is $(1-\beta) \mu_{I}$, and the expected mass of liquidity traders on the exchange is $\alpha_{e} \mathbb{E}\left(Z^{+}+Z^{-}\right)=\alpha_{e} \mu_{z}$. The market maker breaks even on average over both buy and sell orders, ${ }^{16}$ so the spread satisfies

$$
0=-(1-\beta) \mu_{I}(\sigma-S)+\alpha_{e} \mu_{z} S
$$

which implies that

$$
S=\frac{(1-\beta) \mu_{I}}{(1-\beta) \mu_{I}+\alpha_{e} \mu_{z}} \sigma
$$

The dark pool crosses orders at the midmarket price of zero.

Next, I derive the equilibrium mass $\mu_{I}$ of informed traders. Given the value $\sigma$ of information and the exchange spread $S$, the net profit of an informed trader is $\sigma-S$.

\footnotetext{
${ }^{16}$ In this sense, my setting is different from that of Glosten and Milgrom (1985), where the market maker breaks even in expectation on each buy order and each sell order. This modeling choice simplifies the analysis without affecting the main intuition of the paper.
} 
The information-acquisition cost of the marginal for-profit trader, who is indifferent between paying or not paying for information, is also $\sigma-S$. Because all for-profit traders with lower information-acquisition costs become informed, the mass of informed traders in equilibrium is $\bar{\mu} F(\sigma-S)$, by the exact law of large numbers (Sun 2006). We thus have

$$
\mu_{I}=\bar{\mu} F(\sigma-S)=\bar{\mu} F\left(\frac{\alpha_{e} \mu_{z}}{(1-\beta) \mu_{I}+\alpha_{e} \mu_{z}} \sigma\right) .
$$

For any fixed $\beta \geq 0$ and $\alpha_{e}>0$, (4) has a unique solution $\mu_{I} \in(0, \bar{\mu})$.

Finally, I turn to the equilibrium trading strategies. Without loss of generality, I focus on the strategies of buyers. In the main solution step, I calculate the expected payoffs of an informed buyer and a liquidity buyer, on the exchange and in the dark pool. The equilibrium is then naturally determined by conditions characterizing marginal traders who are indifferent between trading on the exchange and in the dark pool.

Suppose that $\alpha_{d}>0$. Because informed buyers trade in the same direction, they have the dark-pool crossing probability of

$$
r^{-}=\mathbb{E}\left[\min \left(1, \frac{\alpha_{d} Z^{-}}{\alpha_{d} Z^{+}+\beta \mu_{I}}\right)\right],
$$

where the denominator and the numerator in the fraction above are the masses of buyers and sellers in the dark pool, respectively. Liquidity buyers, on the other hand, do not observe $v$. If informed traders are buyers, then liquidity buyers have the crossing probability $r^{-}$in the dark pool. If, however, informed traders are sellers, then liquidity buyers have the crossing probability

$$
r^{+}=\mathbb{E}\left[\min \left(1, \frac{\alpha_{d} Z^{-}+\beta \mu_{I}}{\alpha_{d} Z^{+}}\right)\right] .
$$

Obviously, for all $\beta>0$, we have

$$
1>r^{+}>r^{-}>0
$$

Because liquidity buyers assign equal probabilities to the two events $\{v=+\sigma\}$ and $\{v=-\sigma\}$, their dark pool crossing probability $\left(r^{+}+r^{-}\right) / 2$ is greater than informed traders' crossing probability $r^{-}$. In other words, correlated informed orders have a lower execution probability in the dark pool than do relatively uncorrelated liquidity orders.

If the dark pool contains only liquidity orders (i.e., $\beta=0$ ), then any dark-pool buy 
order has the execution probability

$$
\bar{r}=\mathbb{E}\left[\min \left(1, \frac{Z^{-}}{Z^{+}}\right)\right] .
$$

For our purposes, $\bar{r}$ measures the degree to which liquidity orders are balanced. Perfectly balanced liquidity orders correspond to $\bar{r}=1$. Further, as we see shortly, in equilibrium $\alpha_{d}>0$ as long as $\sigma>0$. Thus, the denominator and the numerator on the right-hand side of (5) and (6) are never zero on the equilibrium path, and $r^{-}, r^{+}$, and $\bar{r}$ are well defined.

The expected profits of an informed buyer on the exchange and in the dark pool are, respectively,

$$
\begin{aligned}
& W_{e}=\sigma-S, \\
& W_{d}=r^{-} \sigma .
\end{aligned}
$$

I denote by $c$ the delay cost of a generic liquidity buyer per unit of asset position. This buyer's per-unit net payoffs of deferring trade, trading on the exchange, and trading in the dark pool are, respectively,

$$
\begin{aligned}
X_{0}(c) & =-c, \\
X_{e} & =-S, \\
X_{d}(c) & =-\frac{r^{+}-r^{-}}{2} \sigma-c\left(1-\frac{r^{+}+r^{-}}{2}\right) .
\end{aligned}
$$

The terms on the right-hand side of (13) are the liquidity trader's adverse selection cost and delay cost in the dark pool, respectively. For $\beta>0$, crossing in the dark pool implies a positive adverse selection cost because execution is more likely if a liquidity trader is on the side of the market opposite to that of informed traders. ${ }^{17}$ For $\beta=0$, this adverse-selection cost is zero. For simplicity, in the analysis below the net profits and delay costs of liquidity traders refer to profits and costs per unit of asset, unless otherwise specified. (It is without loss of generality to focus on the venue decision based on per-unit payoffs; see Lemma 1 below.)

\footnotetext{
${ }^{17}$ For example, Sofianos and Xiang (2011) find that dark pools that have higher execution probabilities also have slightly higher adverse selection (that is, they are more "toxic"). Næs and Odegaard (2006) provide anecdotal evidence that filled orders in a dark pool are subject to short-term losses. Mittal (2008) and Saraiya and Mittal (2009) emphasize that short-term adverse selection in dark pools can reduce execution quality of institutional investors. Conrad, Johnson, and Wahal (2003), Brandes and Domowitz (2010), and Domowitz, Finkelshteyn, and Yegerman (2009) examine execution costs in dark pools, although they do not explicitly measure the costs of adverse selection.
} 
From (9) and (12), $W_{e}-X_{e}=\sigma$. For all delay cost $c \leq \sigma$,

$$
W_{d}-X_{d}(c)=\frac{r^{+}+r^{-}}{2} \sigma+c\left(1-\frac{r^{+}+r^{-}}{2}\right) \leq \sigma=W_{e}-X_{e}
$$

That is, provided $c \leq \sigma$, the dark pool is more attractive to liquidity traders than to informed traders, relative to the exchange. In particular, (14) implies that a liquidity trader with a delay cost of $\sigma$ (or a type of $\gamma=1$ ) behaves in the same way as an informed trader. In addition,

$$
X_{d}(c)-X_{0}(c)=-\frac{r^{+}-r^{-}}{2} \sigma+\frac{r^{+}+r^{-}}{2} c .
$$

So a liquidity trader with a type of $\gamma=\left(r^{+}-r^{-}\right) /\left(r^{+}+r^{-}\right)$is indifferent between deferring trade and trading in the dark pool.

Before stating the equilibrium, we emphasize that we do not restrict traders to send orders to only one venue; rather, it is an equilibrium outcome. This result is formally stated in the following lemma.

Lemma 1. If a trader does not delay trades, then it is an equilibrium that he sends his entire order to only one venue with probability one.

The intuition for Lemma 1 is simple. Because each trader has a mass of zero, he has no impact on prices or execution probabilities. Therefore, each trader's optimal strategy is to send his entire order to one venue. (If two venues give equal payoff to a trader, sending the entire order to one venue is also optimal.) While this result relies on the assumption of finite order size per capita, a finite order size is a standard assumption in existing models of dark pools, such as Hendershott and Mendelson (2000), Degryse, Van Achter, and Wuyts (2009), and Buti, Rindi, and Werner (2011a), as well as the classic model of Glosten and Milgrom (1985) and extensions. A finite order size can also be viewed as a substitute for (unmodeled) credit limit or capital constraint. ${ }^{18}$

We are now ready to characterize the equilibrium.

Proposition 1. There exists a unique threshold volatility $\bar{\sigma}>0$ such that:

(1) If $\sigma \leq \bar{\sigma}$, then there exists an equilibrium $\left(\beta=0, \alpha_{d}=\alpha_{d}^{*}, \alpha_{e}=1-\alpha_{d}^{*}\right)$, where

\footnotetext{
${ }^{18}$ If an infinitesimal trader can trade an infinite number of shares per capita, then that trader can become "large" and strictly prefer splitting his order to avoid "price impact" on the exchange. This size-discovery benefit of dark pools is separate from the price-discovery focus of this paper, as we discussed in the introduction. So far, I have not found a tractable model that combines price discovery and size discovery without making additional restrictive assumptions. For example, Ye (2011) models both price discovery and price impact in the Kyle (1985) framework, but he assumes exogenous venue choice of uninformed traders.
} 
$\alpha_{d}^{*} \in(0, G(1)]$ and $\mu_{I}^{*}$ solve

$$
\begin{aligned}
G^{-1}\left(\alpha_{d}\right)(1-\bar{r}) & =\frac{\mu_{I}}{\mu_{I}+\left(1-\alpha_{d}\right) \mu_{z}} \\
\mu_{I} & =\bar{\mu} F\left(\frac{\left(1-\alpha_{d}\right) \mu_{z}}{\mu_{I}+\left(1-\alpha_{d}\right) \mu_{z}} \sigma\right) .
\end{aligned}
$$

(2) If and only if $\sigma>\bar{\sigma}$, there exists an equilibrium $\left(\beta=\beta^{*}, \alpha_{d}=\alpha_{d}^{*}, \alpha_{e}=1-G(1)\right)$, where $\beta^{*}, \alpha_{d}^{*} \in(0, G(1)]$, and $\mu_{I}^{*}$ solve

$$
\begin{aligned}
r^{-} & =1-\frac{(1-\beta) \mu_{I}}{(1-\beta) \mu_{I}+(1-G(1)) \mu_{z}}, \\
\alpha_{d} & =G(1)-G\left(\frac{r^{+}-r^{-}}{r^{+}+r^{-}}\right), \\
\mu_{I} & =\bar{\mu} F\left(\frac{(1-G(1)) \mu_{z}}{(1-\beta) \mu_{I}+(1-G(1)) \mu_{z}} \sigma\right) .
\end{aligned}
$$

The proof of Proposition 1 is provided in Appendix B, but we outline its main intuition here. If the volatility $\sigma$ is sufficiently low, the exchange spread is low; thus, the price-improvement benefit of the dark pool is lower than the cost of execution risk. In this case, informed traders avoid the dark pool (i.e., $\beta=0$ ). The equilibrium is then determined by the marginal liquidity trader who is indifferent between trading on the exchange and trading in the dark pool, as well as by the marginal for-profit trader who is indifferent about whether to acquire the information.

If the volatility $\sigma$ is sufficiently high, informed traders joint liquidity traders in the dark pool to avoid the higher exchange spread. Thus, $\beta \in(0,1)$. In this case, the equilibrium is determined by three indifference conditions. First, informed traders must be indifferent between trading in either venue, as shown in (18). By (14), a liquidity trader with a delay cost of $\sigma$ is also indifferent between the two venues. Thus, $\alpha_{0}+\alpha_{d}=G(1)$ and $\alpha_{e}=1-G(1)$. The second indifference condition (19) then follows from (15). Here, the fraction $\alpha_{0}$ of liquidity traders who delay trade must be strictly positive because informed traders introduce adverse selection into the dark pool. The third condition (20) says that the marginal for-profit trader is indifferent about whether to acquire the information.

Similarly, we can characterize an equilibrium for a market structure in which only the exchange is operating and the dark pool is absent. This exchange-only equilibrium, stated below, may also be interpreted as one in which a dark pool is open but no trader uses it. 
Corollary 1. With only an exchange and no dark pool, there exists an equilibrium in which $\beta^{*}=\alpha_{d}^{*}=0$, and $\mu_{I}^{*}$ and $\alpha_{e}^{*} \in(1-G(1), 1)$ solve

$$
\begin{aligned}
\frac{\mu_{I}}{\mu_{I}+\alpha_{e} \mu_{z}} & =G^{-1}\left(1-\alpha_{e}\right) \\
\mu_{I} & =\bar{\mu} F\left(\frac{\alpha_{e} \mu_{z}}{\mu_{I}+\alpha_{e} \mu_{z}} \sigma\right) .
\end{aligned}
$$

2.2.1 Equilibrium selection. The equilibria characterized in Proposition 1 need not be unique among all equilibria solving (16)-(17) and (18)-(20). For example, under the condition $\sigma \leq \bar{\sigma}$ (derived from condition (B2) in the Appendix), both sides of (16) strictly increase in $\alpha_{d}$. Similarly, both sides of (19) strictly increase in $\alpha_{d}$, and both sides of (21) strictly decrease in $\alpha_{e}$. Thus, given the absence of a single-crossing property, multiple equilibria may arise due to coordination: although no single trader wishes to unilaterally deviate, the collective deviation of a sufficient mass of traders may move the equilibrium. For example, if a sufficiently large mass of liquidity traders are "perturbed" and move from the exchange to the dark pool, this movement may increase the exchange spread so much that those perturbed liquidity traders will stay in the dark pool. ${ }^{19}$ A different equilibrium may emerge. ${ }^{20}$

I use stability as an equilibrium selection criterion, which allows me to compute the comparative statics of the selected equilibria. Among the equilibria characterized by Case 1 of Proposition 1, I select that with the smallest liquidity participation $\alpha_{d}^{*}$ in the dark pool among those with the property that, as $\alpha_{d}$ varies in the neighborhood of $\alpha_{d}^{*}$, the left-hand side of (16) crosses the right-hand side from below. ${ }^{21}$ Under the conditions of Proposition 1, this equilibrium exists and is robust to small perturbations. ${ }^{22}$ Moreover, selecting the stable equilibrium with the smallest $\alpha_{d}^{*}$ provides a conservative

\footnotetext{
${ }^{19}$ In my model, a perturbation of a large mass of liquidity traders into the dark pool needs not increase the execution probability in the dark pool. In Case 1 of Proposition 1, for example, if an extra fraction $\epsilon>0$ of liquidity traders are perturbed into the dark pool, the dark-pool execution probability stays the same, $\bar{r}$, because there are infinitely many infinitesimal traders. In the model of Hendershott and Mendelson (2000), a larger number of uninformed traders in the dark pool implies a higher execution probability because there are finitely many traders.

${ }^{20}$ One condition that guarantees the uniqueness of the equilibrium in Case 1 of Proposition 1 is that the distribution function $G$ of delay costs is linear. With a linear $G$, the condition (B2) in the Appendix is also necessary for the existence of solutions to (16)-(17).

${ }^{21}$ Selecting the stable equilibrium corresponding to the smallest $\alpha_{d}^{*}$ is arbitrary but without loss of generality. As long as the selected equilibrium is stable, comparative statics calculated later follow through.

${ }^{22}$ If, for example, $\alpha_{d}^{*}$ is perturbed to $\alpha_{d}^{*}+\epsilon$ for sufficiently small $\epsilon>0$, then the marginal liquidity trader has a higher cost in the dark pool than on the exchange, and therefore migrates out of the dark pool. Thus, $\alpha_{d}$ is "pushed back" to $\alpha_{d}^{*}$ and the equilibrium is restored. There is a symmetric argument for a small downward perturbation to $\alpha_{d}^{*}-\epsilon$. By contrast, if there is an equilibrium in which, as $\alpha_{d}$ varies, the left-hand side of (16) crosses the right-hand side from above, this equilibrium would not be stable to local perturbations.
} 
estimate of the effect of the dark pool. ${ }^{23}$ Once $\alpha_{d}$ is determined in equilibrium, $\mu_{I}$ and $\beta$ are uniquely determined, too, as shown in the proof of Proposition 1.

Similarly, among equilibria characterized by Case 2 of Proposition 1, I select the one with the smallest liquidity participation $\alpha_{d}^{*}$ in the dark pool among those with the property that, as $\alpha_{d}$ varies in the neighborhood of $\alpha_{d}^{*}$, the left-hand side of (19) crosses the right-hand side from below. In a market without a dark pool (Corollary 1), I select the equilibrium with the largest liquidity participation $\alpha_{e}^{*}$ on the exchange among those with the property that, as $\alpha_{e}$ varies in the neighborhood of $\alpha_{e}^{*}$, the left-hand side of (21) crosses the right-hand side from below. By the argument given for Case 1 of Proposition 1, these selected equilibria exist and are stable.

2.2.2 Discussion. My modeling approach and results complement a few branches of prior literature. First, in prior microstructure models with multiple exchanges, informed traders and liquidity traders tend to cluster by time (Admati and Pfleiderer 1988) or by location (Pagano 1989; Chowdhry and Nanda 1991). As modeled in this paper, however, informed traders endogenously cluster less with liquidity traders in the dark pool than on the exchange because of different execution risks in the dark pool. $^{24}$ Second, my model relies on self-selection, rather than on nonanonymity, to separate, at least partially, informed traders from liquidity traders. Models that rely on nonanonymity (explicitly or implicitly) to filter informed traders include those of Seppi (1990), Benveniste, Marcus, and Wilhelm (1992), Easley, Keifer, and O'Hara (1996), Madhavan and Cheng (1997), Grammig, Schiereck, and Theissen (2001), and Garfinkel and Nimalendran (2003), among others. Third, my study of dark pools differs from existing papers on nondisplayed markets that operate alone, such as Boulatov and George (2013), Hendershott and Jones (2005), and Bloomfield, O'Hara, and Saar (2012).

\subsection{Market characteristics and comparative statics}

I now investigate properties of the equilibria characterized by Proposition 1. Proposition 2 and Proposition 3 below aim to answer two questions:

(1) In a market with a dark pool and an exchange, how do market characteristics vary with volatility $\sigma$ ?

\footnotetext{
${ }^{23}$ If another stable equilibria with a larger $\alpha_{d}^{*}$ exists, using that equilibrium is likely to make my results stronger by increasing the mass of liquidity traders in the dark pool.

${ }^{24}$ In a recent model by Guerrieri and Shimer (2012), investors trade multiple assets with different qualities, but each type of asset only trades in one market; in my model, investors separate with a single asset by trading on different venues.
} 
(2) Given a fixed volatility $\sigma$, how does adding a dark pool affect market behavior?

Proposition 2. In the equilibrium of Proposition 1:

(1) For $\sigma \leq \bar{\sigma}$, the dark pool participation rate $\alpha_{d}$ of liquidity traders, the total mass $\mu_{I}$ of informed traders, and the scaled exchange spread $S / \sigma$ are strictly increasing in $\sigma$. The exchange participation rate $\alpha_{e}=1-\alpha_{d}$ of liquidity traders is strictly decreasing in $\sigma$. Moreover, $\alpha_{d}, \mu_{I}$, and $S$ are continuous and differentiable in $\sigma$.

(2) For $\sigma>\bar{\sigma}$, all of $\mu_{I}, \beta \mu_{I}, r^{+}$, and $S / \sigma$ are strictly increasing in $\sigma$, whereas $\alpha_{d}$ and $r^{-}$are strictly decreasing in $\sigma$. Moreover, $\beta, \alpha_{d}, \mu_{I}, S, r^{+}$, and $r^{-}$are continuous and differentiable in $\sigma$.

In the equilibrium of Corollary $1, \mu_{I}$ and $S / \sigma$ are strictly increasing in $\sigma$, whereas $\alpha_{e}$ is strictly decreasing in $\sigma$. Moreover, $\alpha_{e}, \mu_{I}$, and $S$ are continuous and differentiable in $\sigma$.

Proposition 3. In the equilibria of Proposition 1 and Corollary 1:

(1) For $\sigma \leq \bar{\sigma}$, adding a dark pool strictly reduces the exchange participation rate $\alpha_{e}$ of liquidity traders and the total mass $\mu_{I}$ of informed traders. Adding a dark pool strictly increases the exchange spread $S$ and the total participation rate $\alpha_{e}+\alpha_{d}$ of liquidity traders.

(2) For $\sigma>\bar{\sigma}$, adding a dark pool strictly reduces $\alpha_{e}$. Moreover, adding a dark pool strictly increases the exchange spread $S$ if and only if, in the equilibrium of Proposition 1,

$$
r^{-}<1-\frac{\mu_{I}}{\mu_{I}+\left(1-G\left(1-r^{-}\right)\right) \mu_{z}} .
$$

It is sufficient (but not necessary) for (23) that

$$
G^{\prime \prime}(\gamma) \leq 0 \text { for all } 1-\bar{r} \leq \gamma \leq 1 \text { and } F(c) \rightarrow 1 \text { for all } c>0
$$

We now discuss the intuition and implications of Proposition 2 and Proposition 3. Whereas the equilibria and comparative statics are characterized analytically, the solutions of equilibrium variables are not in closed form. I solve them numerically using the Matlab function fsolve.

2.3.1 Participation rates and exchange spread. The left-hand-side plot of Figure 3 shows the equilibrium participation rates in the exchange and the dark pool. For a small value of information, specifically if $\sigma \leq \bar{\sigma}$, informed traders trade exclusively on the exchange because the exchange spread is smaller than the cost of execution risk in the dark pool. An increase in $\sigma$ widens the exchange spread, encouraging more 
Figure 3: Participation rates and exchange spread

The left-hand side plot shows the equilibrium participation rates $\left(\beta, \alpha_{d}, \alpha_{e}\right)$ in a market with a dark pool. The right-hand-side plot shows the scaled exchange spread $S / \sigma$. In both plots, the vertical dotted line indicates the threshold volatility $\bar{\sigma}$ at which the equilibrium of Proposition 1 changes from Case 1 to Case 2. Model parameters: $\mu_{z}=60, \sigma_{z}=\sqrt{60}, \bar{\mu}=20, Z^{+}$and $Z^{-}$have Gamma distributions with mean 30 and variance $30, G(s)=s / 2$ for $s \in[0,2]$, and $F(s)=1-e^{-s / 2}$ for $s \in[0, \infty)$.
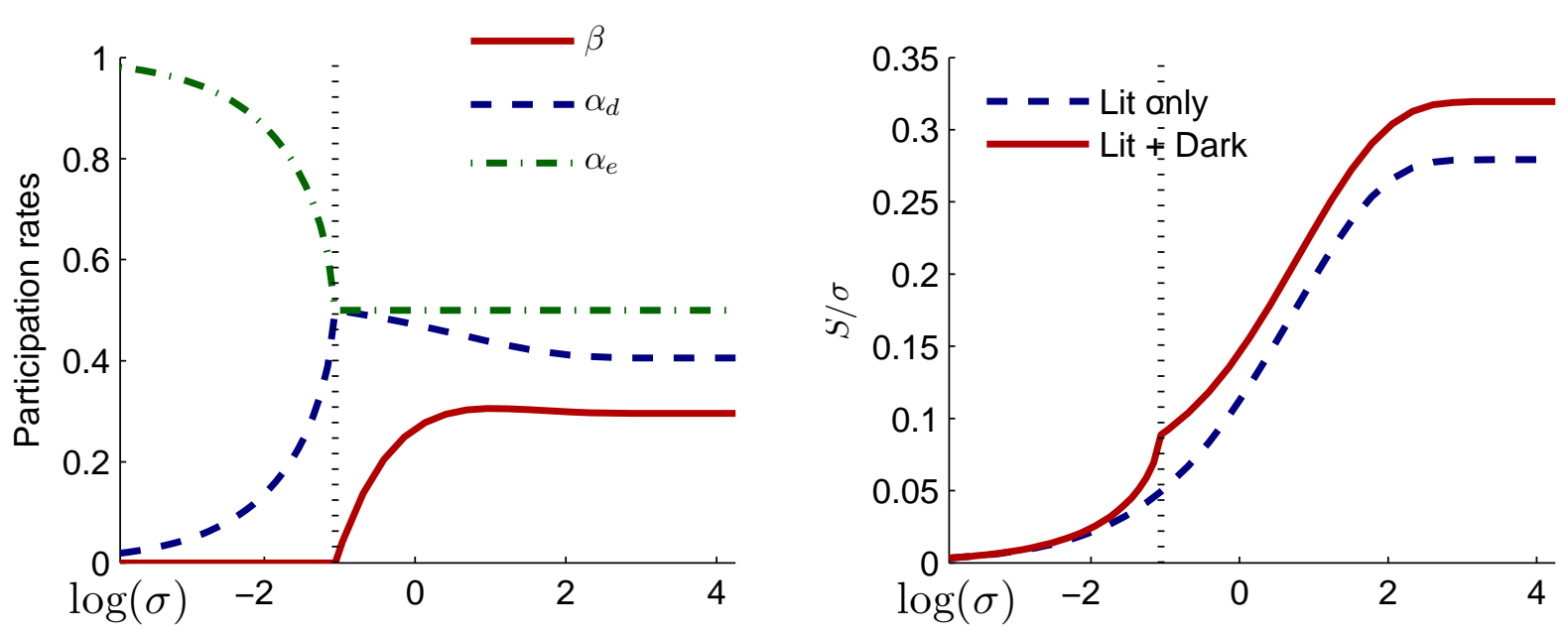

liquidity traders to migrate into the dark pool. For $\sigma>\bar{\sigma}$, informed traders use both venues. Because informed participation in the dark pool introduces adverse selection, liquidity traders with low delay costs migrate out of the dark pool, leading to a decline in their dark pool participation rate $\alpha_{d}$.

We observe that informed dark pool participation rate $\beta$ first increases in volatility $\sigma$ and then decreases. The intuition for this nonmonotonicity is as follows. A higher value of information $\sigma$ encourages additional for-profit traders to become informed. For a low $\beta$, the dark pool execution risk is relatively low, and these additional informed traders prefer the dark pool, raising $\beta$. For sufficiently high $\beta$, however, informed orders cluster on one side of the dark pool and significantly reduce their execution probability. Thus, these additional informed traders send orders to the exchange, reducing $\beta$. Nonetheless, the total quantity $\beta \mu_{I}$ of informed traders in the dark pool is strictly increasing in $\sigma$.

The right-hand-side plot of Figure 3 shows the scaled exchange spread $S / \sigma$. Because a higher value $\sigma$ of information encourages more for-profit traders to become informed, the scaled exchange spread $S / \sigma$ increases in $\sigma$, whether or not a dark pool is present. For $\sigma \leq \bar{\sigma}$, adding a dark pool raises $S / \sigma$ by diverting some liquidity traders, but none of the informed traders, off the exchange. For $\sigma>\bar{\sigma}$, adding a dark pool in this example also increases the scaled spread $S / \sigma$ because the dark pool diverts more 
Figure 4: Expected trading volume on the exchange and in the dark pool

The left-hand plot shows the volume in the two venues and the market share of the dark pool. The right-hand plot shows the dark pool market share as a function of the scaled spread $S / \sigma$. The vertical dotted line corresponds to the threshold volatility $\bar{\sigma}$. Parameters are those of Figure 3.
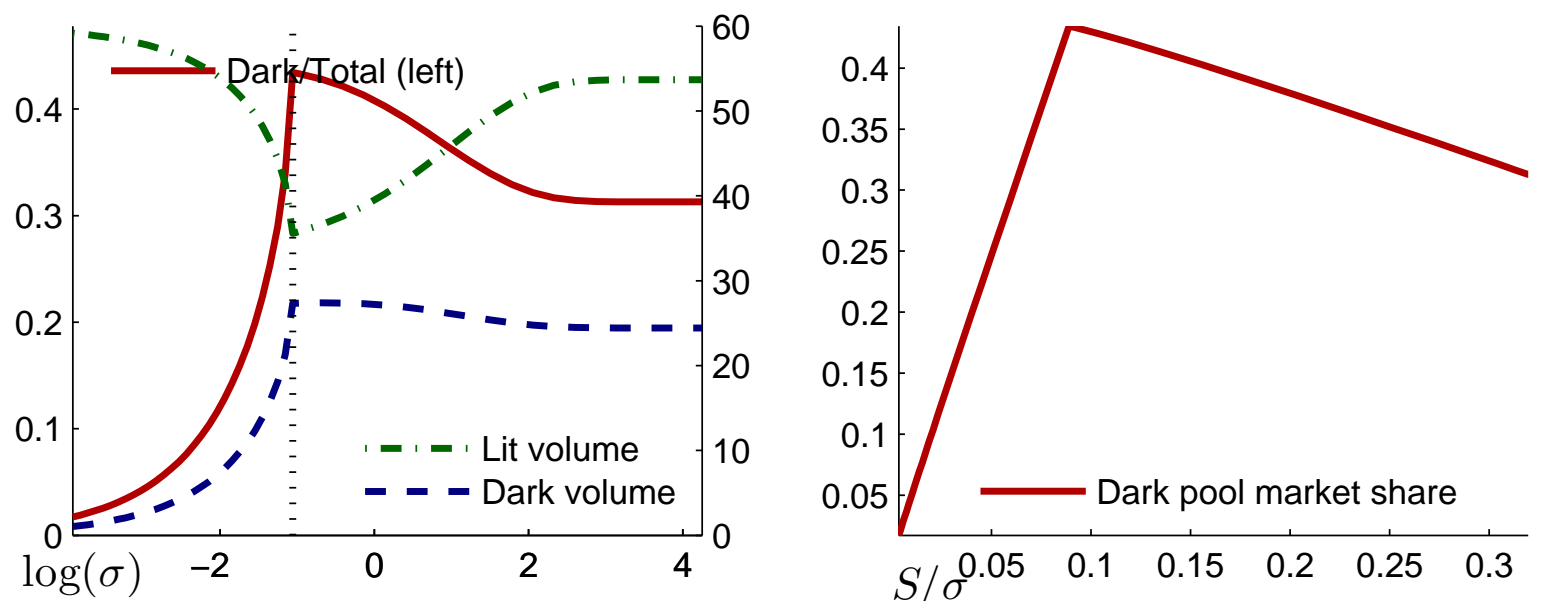

liquidity traders than informed traders.

2.3.2 Dark pool market share. I now calculate the dark pool market share, that is, the proportion of trading volume handled by the dark pool. The market share of the dark pool is a direct empirical measure of dark pool activity. I assume that once the dividend $v$ is announced in period 2, informed traders who have not yet traded leave the market, because they will not be able to trade profitably. When calculating the exchange volume, I also include the transactions of liquidity traders in period 2. Thus, the expected trading volumes in the dark pool, on the exchange, and in both venues are, respectively,

$$
\begin{aligned}
& V_{d}=\beta \mu_{I} r^{-}+\alpha_{d} \mu_{z} \frac{r^{+}+r^{-}}{2}, \\
& V_{e}=(1-\beta) \mu_{I}+\alpha_{e} \mu_{z}+\alpha_{d} \mu_{z}\left(1-\frac{r^{+}+r^{-}}{2}\right)+\alpha_{0} \mu_{z}, \\
& V=V_{e}+V_{d}=\mu_{z}+\mu_{I}\left(1-\beta+\beta r^{-}\right) .
\end{aligned}
$$

Figure 4 illustrates the equilibrium behavior of dark pool market share. The lefthand plot shows that for sufficiently small volatility $(\sigma \leq \bar{\sigma})$, the dark pool market share is increasing in the volatility $\sigma$. This is because in Case 1 of Proposition 1, a higher volatility increases the dark pool volume but reduces the exchange volume. As the volatility $\sigma$ increases beyond $\bar{\sigma}$, however, the exchange volume $V_{e}$ can increase substantially, but the dark pool volume $V_{d}$ may only increase mildly or even decline. 
Thus, the dark-pool market share can decrease in volatility $\sigma$ for sufficiently large $\sigma$, creating a hump-shaped relation between volatility and the dark pool market share. The model also generates a similar relation between the scaled spread $S / \sigma$ and the dark-pool market share $V_{d} / V$, as shown on the right-hand plot of Figure 4 .

\subsection{Price discovery}

Now I turn to price discovery, by which I mean the extent to which the period 1 announcements $\left(P_{1}, V_{b}, V_{s}\right)$ are informative of the fundamental asset value $v$. Because the market maker observes the volume $\left(V_{b}, V_{s}\right)$, the closing price $P_{1}$ is

$$
P_{1}=\mathbb{E}\left[v \mid V_{b}, V_{s}\right]
$$

Because $v$ is binomially distributed, its conditional distribution after period 1 trading is completely determined by its conditional expectation

$$
\mathbb{E}\left[v \mid P_{1}, V_{b}, V_{s}\right]=\mathbb{E}\left[\mathbb{E}\left[v \mid V_{b}, V_{s}\right] \mid P_{1}\right]=P_{1} .
$$

That is, all period 1 public information that is relevant for the asset value $v$ is conveyed by the closing price $P_{1}$. As we will make precise shortly, the "closer" is $P_{1}$ to $v$, the better is price discovery.

Clearly, $P_{1}$ is uniquely determined by the log likelihood ratio

$$
R_{1}=\log \frac{\mathbb{P}\left(v=+\sigma \mid V_{b}, V_{s}\right)}{\mathbb{P}\left(v=-\sigma \mid V_{b}, V_{s}\right)}=\log \frac{\phi\left(Z^{+}=\frac{1}{\alpha_{e}}\left[V_{b}-(1-\beta) \mu_{I}\right]\right) \cdot \phi\left(Z^{-}=\frac{1}{\alpha_{e}} V_{s}\right)}{\phi\left(Z^{+}=\frac{1}{\alpha_{e}} V_{b}\right) \cdot \phi\left(Z^{-}=\frac{1}{\alpha_{e}}\left[V_{s}-(1-\beta) \mu_{I}\right]\right)},
$$

where $\phi$ is the probability density function of $Z^{+}$and $Z^{-}$. We have also used the fact that the prior distribution $\mathbb{P}(v=+\sigma)=\mathbb{P}(v=-\sigma)=0.5$.

Given $R_{1}$, the market maker sets the period 1 closing price

$$
P_{1}=\frac{e^{R_{1}}-1}{e^{R_{1}}+1} \sigma .
$$

Without loss of generality, I condition on $v=+\sigma$ and consider price discovery to be unambiguously "improved" if the probability distribution of $R_{1}$ is "increased," in the sense of first-order stochastic dominance. Complete revelation of $v=+\sigma$ corresponds to $R_{1}=\infty$ almost surely.

Using the $\log$ likelihood ratio $R_{1}$, we can also calculate the scaled root-mean-squared 
error (RMSE):

$$
\frac{\sqrt{\mathbb{E}\left[\left(v-P_{1}\right)^{2} \mid v=\sigma\right]}}{\sigma}=\sqrt{\mathbb{E}\left[\frac{4}{\left(e^{R_{1}}+1\right)^{2}} \mid v=\sigma\right]} .
$$

Here, we scale the RMSE by $\sigma$ to focus on the proportional pricing error, which is always between 0 and 1, rather than dollar pricing error, which, as $\sigma \rightarrow \infty$, can in principal become arbitrarily large.

In general, we need to know the functional form of the density $\phi(\cdot)$ in order to calculate $R_{1}, P_{1}$, and the scaled RMSE. To make the intuition more explicit, and without loss of generality, I take an example in which the density $\phi(\cdot)$ is approximated by $\mathcal{N}\left(0.5 \mu_{z}, 0.5 \sigma_{z}^{2}\right)$ when $\mu_{z}$ and $\sigma_{z}^{2}$ are sufficiently large. ${ }^{25}$ Substituting the normal density function into (30), we can approximate $R_{1}$ by

$$
R_{1}^{\text {normal }}=\frac{2(1-\beta) \mu_{I}}{\alpha_{e}^{2} \sigma_{z}^{2}}\left(V_{b}-V_{s}\right)
$$

which is the counterpart of $R_{1}$ under the normal distribution. Given that $v=+\sigma$, $V_{b}-V_{s}$ has a distribution close to that of $\mathcal{N}\left((1-\beta) \mu_{I}, \alpha_{e}^{2} \sigma_{z}^{2}\right)$, so $R_{1}$ has a distribution close to that of

$$
\mathcal{N}\left(2\left(\frac{(1-\beta) \mu_{I}}{\alpha_{e} \sigma_{z}}\right)^{2}, 4\left(\frac{(1-\beta) \mu_{I}}{\alpha_{e} \sigma_{z}}\right)^{2}\right) \sim \mathcal{N}\left(2 I\left(\beta, \alpha_{e}\right)^{2}, 4 I\left(\beta, \alpha_{e}\right)^{2}\right)
$$

where

$$
I\left(\beta, \alpha_{e}\right) \equiv \frac{(1-\beta) \mu_{I}}{\alpha_{e} \sigma_{z}}
$$

is the "signal-to-noise" ratio, which is the mass of informed orders on the exchange ("signal") divided by the standard deviation of the imbalance of liquidity orders on the exchange ("noise").

Clearly, $I\left(\beta, \alpha_{e}\right)$ is increasing in the scaled exchange spread $S / \sigma=\frac{(1-\beta) \mu_{I} / \alpha_{e}}{(1-\beta) \mu_{I} / \alpha_{e}+\mu_{z}}$. Combining this fact with Proposition 3 , we can immediately infer the effect of the dark pool on price discovery, measured by $I\left(\beta, \alpha_{e}\right)$. In addition, under the normal approximation, we can show analytically that a higher signal-to-noise ratio $I\left(\beta, \alpha_{e}\right)$ corresponds to a lower scaled RMSE. This result is summarized by the following proposition.

\footnotetext{
${ }^{25}$ We can show this approximation as follows. Fix a small $\delta>0$ such that $m=\mu_{z} / \delta$ is an integer. By infinite divisibility, $Z^{+}$can be represented as the sum $\sum_{i=1}^{m} Z_{i m}^{+}$, where $\left\{Z_{i m}^{+}\right\}$are i.i.d. random variables with mean $\delta$ and variance $\delta \sigma_{z}^{2} / \mu_{z}$. Fixing $\delta$, the central limit theorem implies that the distribution of $Z^{+}$is approximately normal when $m$ is large, that is, when $\mu_{z}$ and $\sigma_{z}^{2}$ are large.
} 
Figure 5: Two measures of price discovery, as functions of volatility $\sigma$ The left-hand plot shows the signal-to-noise ratio, and the right-hand plot shows scaled RMSE. The true asset value is $v=\sigma$, and other model parameters are those of Figure 3.
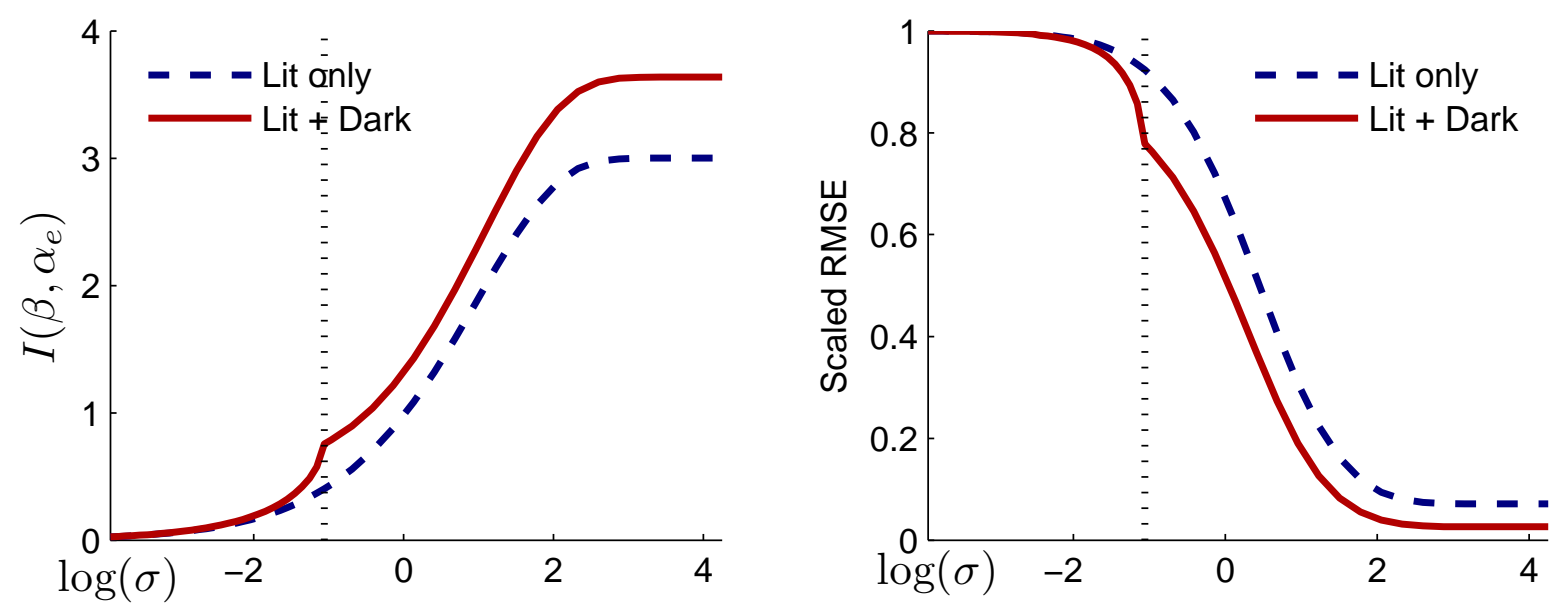

Proposition 4. Under the normal approximation of $\phi$, in the equilibria of Proposition 1 and Corollary 1:

(1) For $\sigma \leq \bar{\sigma}$, adding a dark pool strictly increases the signal-to-noise ratio $I\left(\beta, \alpha_{e}\right)$ on the exchange and strictly decreases the scaled RMSE $\sqrt{\mathbb{E}\left[4\left(e^{R_{1}}+1\right)^{-2} \mid v=\sigma\right]}$.

(2) For $\sigma>\bar{\sigma}$, if the condition specified in Case 2 of Proposition 3 holds, adding a dark pool strictly increases the signal-to-noise ratio $I\left(\beta, \alpha_{e}\right)$ on the exchange and strictly decreases the scaled RMSE $\sqrt{\mathbb{E}\left[4\left(e^{R_{1}}+1\right)^{-2} \mid v=\sigma\right]}$.

Figure 5 plots the two measures of price discovery, signal-to-noise ratio and scaled RMSE, as functions of $\sigma$, with and without a dark pool. We can see that the addition of a dark pool raises the signal-to-noise ratio and reduces the scaled RMSE. For a $\sigma$ close to zero, few for-profit traders become informed. In this case, the exchange order flow contains little information about $v$, and the market maker's pricing error is close to $100 \%$ of the volatility, regardless of whether the dark pool is present. For a large $\sigma$, however, many for-profit traders become informed, and the dark pool starts to concentrate informed traders on the exchange. In this case, adding a dark pool significantly reduces the market maker's percent pricing error from $7.1 \%$ to $2.6 \%$.

So far, we have used a single statistic (the signal-to-noise ratio or the scaled RMSE) to capture the average effect of the dark pool for price discovery. The realized exchange closing price $P_{1}$, however, also incorporates the unexpected order imbalance of the liquidity traders. My next step is to examine the probability distribution of the realized 
Figure 6: Probability density functions of $R_{1}$ and $\left|P_{1}-\sigma\right| / \sigma$, with and without a dark pool The distributions are calculated by setting $v=\bar{\sigma}$ and drawing $2 \times 10^{7}$ realizations of $Z^{+}$and $Z^{-}$. The model parameters are those of Figure 3.
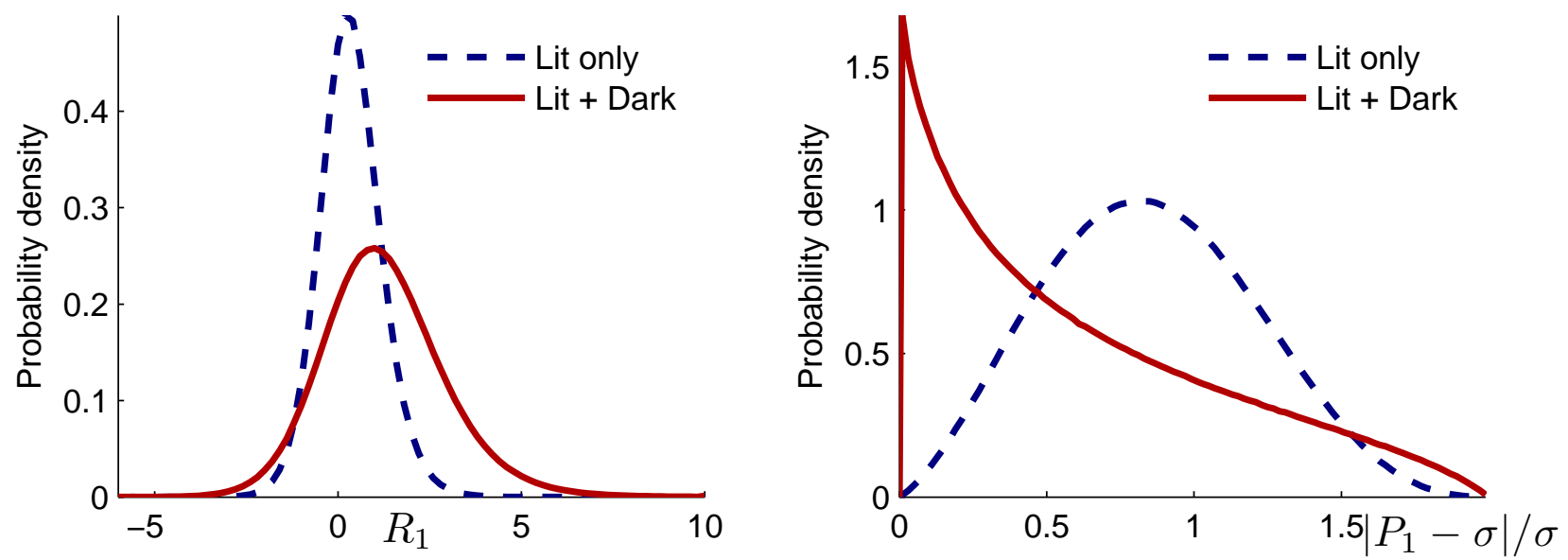

$\log$ likelihood ratio, $R_{1}$, and the realized absolute pricing error scaled by $\sigma$ :

$$
\frac{\left|P_{1}-\sigma\right|}{\sigma}=\frac{2}{\left|e^{R_{1}}+1\right|} .
$$

This exercise would shed light on the following question: does the dark pool improve price discovery path-by-path?

Figure 6 plots the probability density functions of $R_{1}$ and $\left|P_{1}-\sigma\right| / \sigma$ with and without a dark pool. The value $\sigma$ of information is set as the threshold value $\bar{\sigma}$, so that $\beta=0$ in the equilibria with a dark pool as well as the equilibria without a dark pool. We see that on average, the addition of a dark pool shifts the probability density of $R_{1}$ to the right and the probability density of $\left|P_{1}-\sigma\right| / \sigma$ to the left. Price discovery is thus improved in expectation, as we have seen in Figure 5. Nonetheless, the dark pool also "fattens" the left tail of $R_{1}$ and the right tail of $\left|P_{1}-\sigma\right| / \sigma$, harming price discovery in the extreme and unlikely events that $R_{1}$ becomes sufficiently negative (or $P_{1}$ becomes sufficiently close to $\left.-\sigma\right)$. This tail effect has a simple intuition. When the trading interests of liquidity traders are sufficiently large and opposite in direction to the informed - such as under a large liquidity shock - the exchange order flow gives the market maker an incorrect inference regarding the trading direction of the informed traders. Conditional on an incorrect inference, the larger is the signal-to-noise ratio, the larger is the realized pricing error. Therefore, by increasing the signal-to-noise ratio, the dark pool can harm price discovery in those tail events, while improving price discovery in most cases and on average. 
A natural next question is whether, on average, adding a dark pool always improves price discovery. The answer is "not always," as I illustrate through an example. My objectives of constructing this example are twofold. First, the example sharpens my theory by illustrating the limitations of the self-selection mechanism. Second, I use this example to reiterate that my results should not be taken as unqualified "favoring" of dark pools as a policy recommendation. I put this example in the Online Appendix to preserve space, but let me outline the intuition for the construction.

Recall that the key mechanism of my model is self-selection: once a dark pool is introduced, under certain conditions liquidity traders migrate to the dark pool more than informed do, improving price discovery on the exchange. If a dark pool were to harm price discovery, this self-selection mechanism needs to be shut down or limited. A convenient way to limit self-selection is to assume a discontinuous (e.g., binomial) distribution of delay costs of liquidity traders. This discontinuity creates the possibility of a "corner solution," in which one type of liquidity traders is "stuck" on the exchange and the other type is "stuck" in the dark pool (or delays trades). At a corner solution like this, introducing the dark pool moves informed traders, but not liquidity traders, off the exchange and harms price discovery. At a technical level, this construction is analogous to "endogenizing" the key modeling assumption of Ye (2011) that liquidity traders have exogenously assigned trading venues. That said, to the extent that several recent empirical studies have found evidence consistent with the predictions of my model (see Section 4), but not those of Ye (2011), the self-selection mechanism of my model is relevant and important in reality.

\section{Robustness}

In this subsection I examine the robustness of my results in Section 2 in two aspects: dark-pool volume reporting and information horizon.

\subsection{Reporting dark pool volume}

So far, the market maker does not use the dark-pool trading volume in determining the closing price $P_{1}$. In practice, dark pool volumes are reported fairly quickly, if not always in real time. ${ }^{26}$ Suppressing the information contained in dark pool trading volume clearly understates the benefit of the dark pool for price discovery. The next

\footnotetext{
${ }^{26}$ In the United States, dark pools are required to report trades as soon as possible, but within 90 seconds; currently more than $99 \%$ of trades are reported within 30 seconds (see Securities and Exchange Commission 2010). In Canada, dark pool trades are reported in real time.
} 
result shows that the dark-pool volume, combined with the exchange buy and sell volumes, can reveal almost all information of informed traders. Intuitively, once the dark pool reports volume, the market maker can infer three unknown variables $\left(v, Z^{+}\right.$, and $Z^{-}$) from the three observed volumes (exchange buy volume, exchange sell volume, and the dark pool volume).

Proposition 5. Suppose that the dark pool reports its trading volume immediately after period 1 trading. Then, there exists null sets (i.e., sets with measures of zero) $\mathcal{C}_{\sigma} \in \mathbb{R}_{+}$and $\mathcal{C}_{z} \in \mathbb{R}_{+}^{2}$, such that if $\sigma$ is not in $\mathcal{C}_{\sigma}$ and $\left(Z^{+}, Z^{-}\right)$is not in $\mathcal{C}_{z}$, the trading volumes on the exchange and the dark pool perfectly reveal $v$.

\subsection{Information horizon}

So far, information in Section 2 is short term: it is revealed immediately in the beginning of period 2. In this section, I study the effect of adding a dark pool for price discovery if private information is long lived. To be conservative, I assume that the dark pool does not report volume after period 1. Proposition 5 suggests that shutting down the dark pool volume is likely to understate my results.

As in Section 2, the traded asset has the value $v=\sigma$ or $v=-\sigma$ with equal probabilities, and there are two trading periods, $t \in\{1,2\}$. The main difference from Section 2 is that the private information about $v$ can potentially live for two periods. Specifically, $v$ is announced at the beginning of period 2 with probability $\pi \in[0,1]$, and is announced at the end of period 2 (i.e., after period 2 trading) with probability $1-\pi$. The precise timing of the announcement is unknown to anyone, including the informed traders. The announcement time is independent of all else in the model. We can interpret $\pi$ as the horizon of private information; the smaller is $\pi$, the longer is the information horizon.

The possibility of long-term information enriches and complicates the equilibrium analysis. The modeling choice of this section is to introduce information horizon while keeping as many elements of Section 2 as possible. To do this, I make the following assumptions:

Assumption 1. (1) The dark pool does not report period 1 trading volume by the beginning of period 2 .

(2) The dark pool is closed in period 2, and traders who wish to trade in period 2 send market orders to the exchange.

(3) In period 1 , the exchange market maker determines in advance a period 2 trading fee $S_{2}>0$. In period 2, if no information is announced before trading, the market 
maker executes all orders at the price $P_{1}$ but charges the fee $S_{2}$ per share. If $v$ is announced at the beginning of period 2 , the market maker executes all orders at the price $v$ at no fee.

Clearly, Part 1 of Assumption 1 shuts down learning by dark-pool volume and understates the effect of dark pools on price discovery. Although Parts 2 and 3 of Assumption 1 can appear restrictive, they isolate the effect of information horizon in a simple way: conditional on $\pi=1$ (i.e., short-lived information), the model of this section reduces to that in Section 2. The trading fee $S_{2}$ charged in period 2 is analogous to the spread $S$ in period 1.

The other elements of the model, including the mass of informed traders, the mass and direction of liquidity traders, and their costs of information acquisition and delay, are the same as in Section 2. I also keep all endogenous variables of Section 2, including the traders' strategy variables $\left(\beta, \alpha_{0}, \alpha_{d}, \alpha_{d}\right)$, the period 1 exchange spread $S$, and the dark-pool crossing probabilities $\left(r^{+}, r^{-}\right)$.

To characterize the equilibrium, I work backward in time. Given traders' strategies, the market maker sets the fee $S_{2}$ such that he breaks even in expectation; that is, the total expected trading fee collected should be equal to the expected adverse-selection cost. Because the mass of informed traders who fail to trade in the dark pool is $\beta \mu_{I}\left(1-r^{-}\right)$, the expected adverse-selection cost of the market maker in period 2 is

$$
\begin{aligned}
& \mathbb{E}\left[\beta \mu_{I}\left(1-r^{-}\right)\left(\sigma-P_{1}\right) \cdot \mathbb{I}_{v=+\sigma}+\beta \mu_{I}\left(1-r^{-}\right)\left(\sigma+P_{1}\right) \cdot \mathbb{I}_{v=-\sigma}\right] \\
= & \beta \mu_{I}\left(1-r^{-}\right)\left[0.5\left(\sigma-\mathbb{E}\left[P_{1} \mid v=\sigma\right]\right)+0.5\left(\sigma+\mathbb{E}\left[P_{1} \mid v=-\sigma\right]\right)\right] \\
= & \beta \mu_{I}\left(1-r^{-}\right)\left(\sigma-\mathbb{E}\left[P_{1} \mid v=\sigma\right]\right),
\end{aligned}
$$

where the last equality follows by symmetry.

If $v=\sigma$, the expected mass of liquidity buyers who fail to cross in the dark pool is

$$
\alpha_{d} \mathbb{E}\left[Z^{+}\left(1-\min \left(1, \frac{\alpha_{d} Z^{-}}{\alpha_{d} Z^{+}+\beta \mu_{I}}\right)\right)\right] \equiv \alpha_{d} \mu_{z}^{-},
$$

and the expected mass of liquidity sellers who fail to cross in the dark pool is

$$
\alpha_{d} \mathbb{E}\left[Z^{-}\left(1-\min \left(1, \frac{\alpha_{d} Z^{+}+\beta \mu_{I}}{\alpha_{d} Z^{-}}\right)\right)\right] \equiv \alpha_{d} \mu_{z}^{+} .
$$

Thus, if $v=\sigma$ the total expected mass of liquidity traders who trade in period 2 is

$$
\alpha_{0} \mathbb{E}\left[Z^{+}+Z^{-}\right]+\alpha_{d} \mu_{z}^{-}+\alpha_{d} \mu_{z}^{+}=\alpha_{0} \mu_{z}+\alpha_{d}\left(\mu_{z}^{-}+\mu_{z}^{+}\right) .
$$


A symmetric calculation shows that, if $v=-\sigma$, the expected mass of liquidity traders in period 2 is also $\alpha_{0} \mu_{z}+\alpha_{d}\left(\mu_{z}^{-}+\mu_{z}^{+}\right)$. Therefore, to break even in expectation, the period 2 trading fee must be

$$
S_{2}=\frac{\beta \mu_{I}\left(1-r^{-}\right)}{\beta \mu_{I}\left(1-r^{-}\right)+\alpha_{0} \mu_{z}+\alpha_{d}\left(\mu_{z}^{-}+\mu_{z}^{+}\right)}\left(\sigma-\mathbb{E}\left[P_{1} \mid v=\sigma\right]\right) .
$$

Again, the fee $S_{2}$ is levied only if $v$ is not announced at the beginning of period 2 .

We now turn to the profits of traders. Recall that a liquidity trader of type $\gamma$ has the delay cost of $\gamma \sigma$. Thus, the per-unit profits of this liquidity trader under the three strategies are defined similarly as in Section 2:

$$
\begin{aligned}
X_{e} & =-S \\
X_{0}(\gamma) & =-\gamma \sigma-(1-\pi) S_{2}, \\
X_{d}(\gamma) & =-\frac{r^{+}-r^{-}}{2} \sigma-\left(1-\frac{r^{+}+r^{-}}{2}\right)\left(\gamma \sigma+(1-\pi) S_{2}\right) .
\end{aligned}
$$

For exposition simplicity in this section, I let $\gamma_{d}$ (resp. $\gamma_{0}$ ) be the highest (i.e., most impatient) type of liquidity trader who sends orders to the dark pool (resp. delays trade) in period 1. By construction,

$$
\alpha_{0}=G\left(\gamma_{0}\right), \quad \alpha_{d}=G\left(\gamma_{d}\right)-G\left(\gamma_{0}\right), \quad \alpha_{e}=1-G\left(\gamma_{d}\right)
$$

An informed buyer's profits for sending the order to the exchange and to the dark pool are, respectively,

$$
\begin{aligned}
& W_{e}=\sigma-S \\
& W_{d}=r^{-} \sigma+\left(1-r^{-}\right)(1-\pi)\left(\sigma-\mathbb{E}\left[P_{1} \mid v=\sigma\right]-S_{2}\right) .
\end{aligned}
$$

As in Section 2, it is without loss of generality to focus on the case of $v=+\sigma$.

The equilibrium with long-term information can be characterized in a similar way as in Section 2. If $\beta=0$, then $\alpha_{0}=0$. If $\beta>0$, then $\alpha_{0}$ can be zero or positive. Intuitively, if $\beta>0$ and $\pi<1$, even a liquidity trader with a zero delay cost would face a positive cost in period 2 (recall that $\left.X_{0}(\gamma)=-\gamma \sigma-(1-\pi) S_{2}\right)$. This liquidity trader may prefer the dark pool to delaying trade. Thus, there are three possible cases of equilibrium, as stated in the next proposition.

Proposition 6. In a market with an exchange and a dark pool, there are three possible cases of equilibrium. 
(1) An equilibrium that has $\beta=0, \alpha_{d}>0$ and $\alpha_{0}=0$ is characterized by the solution $\left(\alpha_{e}^{*}, \mu_{I}^{*}\right)$ to the following equations

$$
\begin{aligned}
\frac{\mu_{I}}{\mu_{I}+\alpha_{e} \mu_{z}} & =(1-\bar{r}) G^{-1}\left(1-\alpha_{e}\right), \\
\mu_{I} & =\bar{\mu} F\left(\frac{\alpha_{e} \mu_{z}}{\mu_{I}+\alpha_{e} \mu_{z}} \sigma\right),
\end{aligned}
$$

subject to the incentive-compatibility condition $W_{e}>W_{d}$, i.e.,

$$
\frac{\alpha_{e} \mu_{z}}{\mu_{I}+\alpha_{e} \mu_{z}} \sigma>r^{-} \sigma+\left(1-r^{-}\right)(1-\pi)\left(\sigma-\mathbb{E}\left[P_{1} \mid v=\sigma\right]\right)
$$

(2) An equilibrium that has $\beta>0, \alpha_{d}>0$ and $\alpha_{0}>0$ is characterized by the indifference conditions $W_{e}=W_{d}, X_{0}\left(\gamma_{0}\right)=X_{d}\left(\gamma_{0}\right)$, and $X_{d}\left(\gamma_{d}\right)=X_{e}$. That is, the equilibrium is given by the solution $\left(\beta^{*}, \mu_{I}^{*}, \gamma_{d}^{*}, \gamma_{0}^{*}\right)$ to the equations

$$
\begin{aligned}
\frac{\alpha_{e} \mu_{z}}{(1-\beta) \mu_{I}+\alpha_{e} \mu_{z}} \sigma & =r^{-} \sigma+\left(1-r^{-}\right)(1-\pi)\left(\sigma-\mathbb{E}\left[P_{1} \mid v=\sigma\right]-S_{2}\right), \\
\mu_{I} & =\bar{\mu} F\left(\frac{\alpha_{e} \mu_{z}}{(1-\beta) \mu_{I}+\alpha_{e} \mu_{z}} \sigma\right), \\
\frac{(1-\beta) \mu_{I}}{(1-\beta) \mu_{I}+\alpha_{e} \mu_{z}} \sigma & =\frac{r^{+}-r^{-}}{2} \sigma+\left(1-\frac{r^{+}+r^{-}}{2}\right)\left(\gamma_{d} \sigma+(1-\pi) S_{2}\right), \\
\frac{r^{+}-r^{-}}{r^{+}+r^{-}} \sigma & =\gamma_{0} \sigma+(1-\pi) S_{2} .
\end{aligned}
$$

(3) An equilibrium that has $\beta>0, \alpha_{d}>0$ and $\alpha_{0}=0$ are characterized by the solution $\left(\beta^{*}, \mu_{I}^{*}, \gamma_{d}^{*}\right)$ to equations (51), (52) and (53), subject to the incentive-compatibility condition

$$
\frac{r^{+}-r^{-}}{r^{+}+r^{-}} \sigma<(1-\pi) S_{2}
$$

The equilibrium without the dark pool can be characterized in a similar way. To conserve space I put it in Section B.8.

I now discuss some of the comparative statics with respect to the information horizon $\pi$. Whereas the equilibrium is characterized analytically, the solutions are solved numerically using the Matlab function fsolve. It is desirable to prove the comparative statics in closed form, but the comparative statics of Proposition 6 are not as tractable as those of Proposition 1, so I resort to numerical illustrations. As in any numerical exercise, I cannot claim that the results below apply to all parameter values. Nonetheless, these illustrations reveal a number of interesting properties of informed trading and price discovery, not all of which are obvious ex ante. 
Figure 7 plots the mass of informed traders in the dark pool, $\beta \mu_{I}$, the fraction of liquidity traders on the exchange, $\alpha_{e}$, and the scaled exchange spread in period $1, S / \sigma$. (For the case with no dark pool, I plot the mass of informed traders who delay trading.) This plot reveals two interesting patterns. First, without a dark pool, informed traders send all orders to the exchange, regardless of the information horizon. Liquidity traders' strategies and the exchange spread are invariant to information horizon $\pi$ as well. Intuitively, although information may not be announced immediately, the competition among informed traders will drive the price $P_{1}$ close to $v$. Effectively, information will be revealed by order flows, so all informed traders use the exchange immediately. This intuition is consistent with the result of Holden and Subrahmanyam (1992) that even long-term information can be revealed in a very short time by informed traders who compete aggressively.

The second observation from Figure 7 is that adding a dark pool encourages informed traders and liquidity traders to move away from the exchange, widening the spread. Somewhat surprisingly, the longer the information horizon (i.e., the smaller is $\pi$ ), the fewer informed traders who go to the dark pool. This result may appear counterintuitive at first: why don't informed traders use the dark pool more for longerlived information? The reason is the following. The longer is the information horizon (i.e., a smaller $\pi$ ), the less likely is the market maker to set a zero spread in period 2. Anticipating a smaller decrease in trading cost in period 2, and because of delay cost, more liquidity traders use the exchange in period 1. Consequently, the exchange spread $S / \sigma$ shrinks, encouraging more informed traders to move from the dark pool to the exchange. This result is opposite to that of Hendershott and Mendelson (2000), where long-lived information implies that all informed traders use the dark pool, as well as a higher exchange spread. ${ }^{27}$

As in Section 2, we use the signal-to-noise ratio, $I\left(\beta, \alpha_{e}\right)=(1-\beta) \mu_{I} /\left(\alpha_{e} \mu_{z}\right)$, and scaled root-mean-squared error (RMSE), $\sqrt{\mathbb{E}\left[\left(P_{1}-v\right)^{2} \mid v=\sigma\right]} / \sigma$, to measure price discovery at the end of period 1 . Figure 8 plots the two measures as functions of $\pi$, with and without a dark pool. Without a dark pool, in equilibrium all informed

\footnotetext{
${ }^{27}$ See Proposition 11 of HM. The intuition of HM's result is as follows. (For simplicity, let us drop dealers' fixed costs and inventory costs in HM's model.) In HM, a shorter information horizon implies that all informed traders go to the exchange. Because traders generate liquidity externality in the dark pool (i.e., "liquidity begets liquidity"), the more informed traders use the exchange, the lower is the dark pool volume, and the higher is the exchange volume. Because the exchange prices are fixed over time, the exchange spread is proportional to the ratio between the buy-less-sell exchange order imbalance, say BuyLessSell, and the total buy-plus-sell exchange volume, say BuyPlusSell. The numerator BuyLessSell is fixed (the dark pool has no effect on total order imbalance), and the denominator BuyPlusSell is higher if the exchange volume is higher, that is, if the information is short-lived. Therefore, in HM, short lived information implies that all informed traders go to the exchange and a narrower exchange spread, opposite of the pattern shown in Figure 7.
} 
Figure 7: Strategies as functions of information horizon $\pi$

Left: Mass of informed traders in the dark pool, $\beta \mu_{I}$ (if there is no dark pool, I plot the mass of informed traders who delay trade). Middle: Fraction of liquidity traders on the exchange, $\alpha_{e}$. Right: Scaled exchange spread in period $1, S / \sigma$. In this plot, $\sigma=\exp (1)$, and other model parameters are the same as in Figure 3.
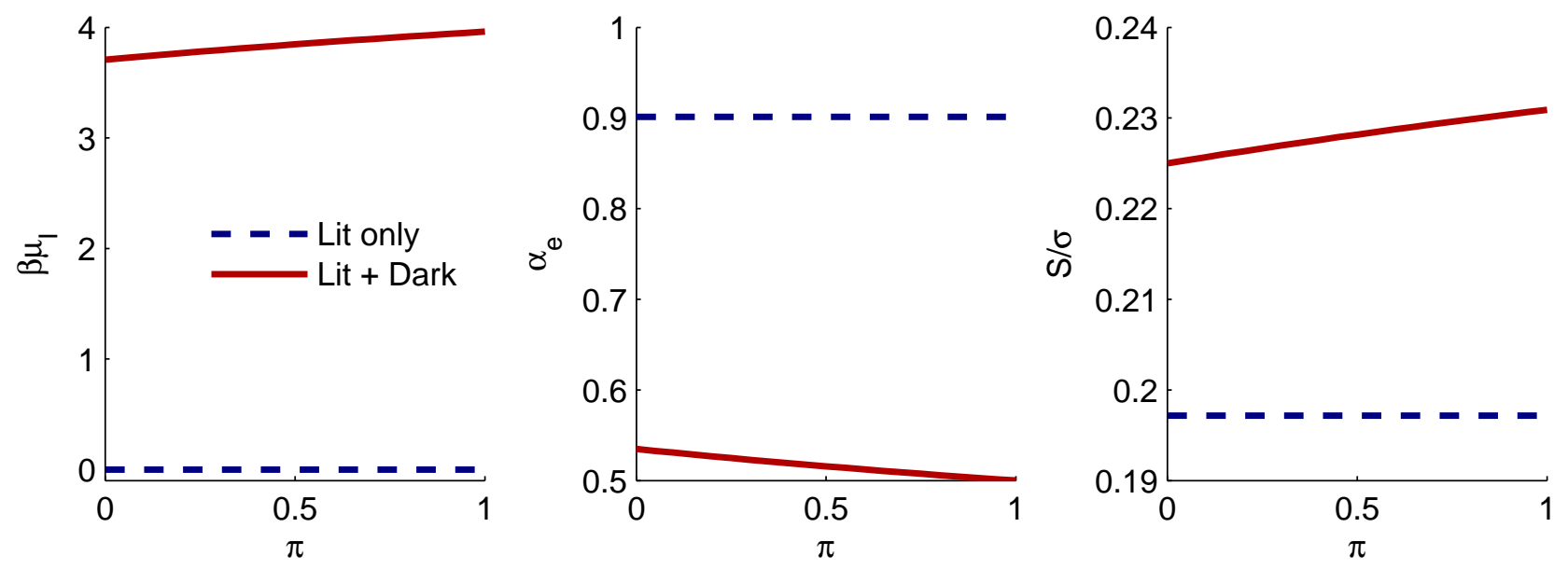

Figure 8: Price-discovery measures as functions of information horizon $\pi$

The left-hand plot shows the signal-to-noise ratio, $I\left(\beta, \alpha_{e}\right)$. The right-hand plot shows the scaled root-mean-squared error, $\sqrt{\mathbb{E}\left[\left(P_{1}-v\right)^{2} \mid v=\sigma\right]} / \sigma$. In both plots, $\sigma=\exp (1)$, and other model parameters are the same as in Figure 3.
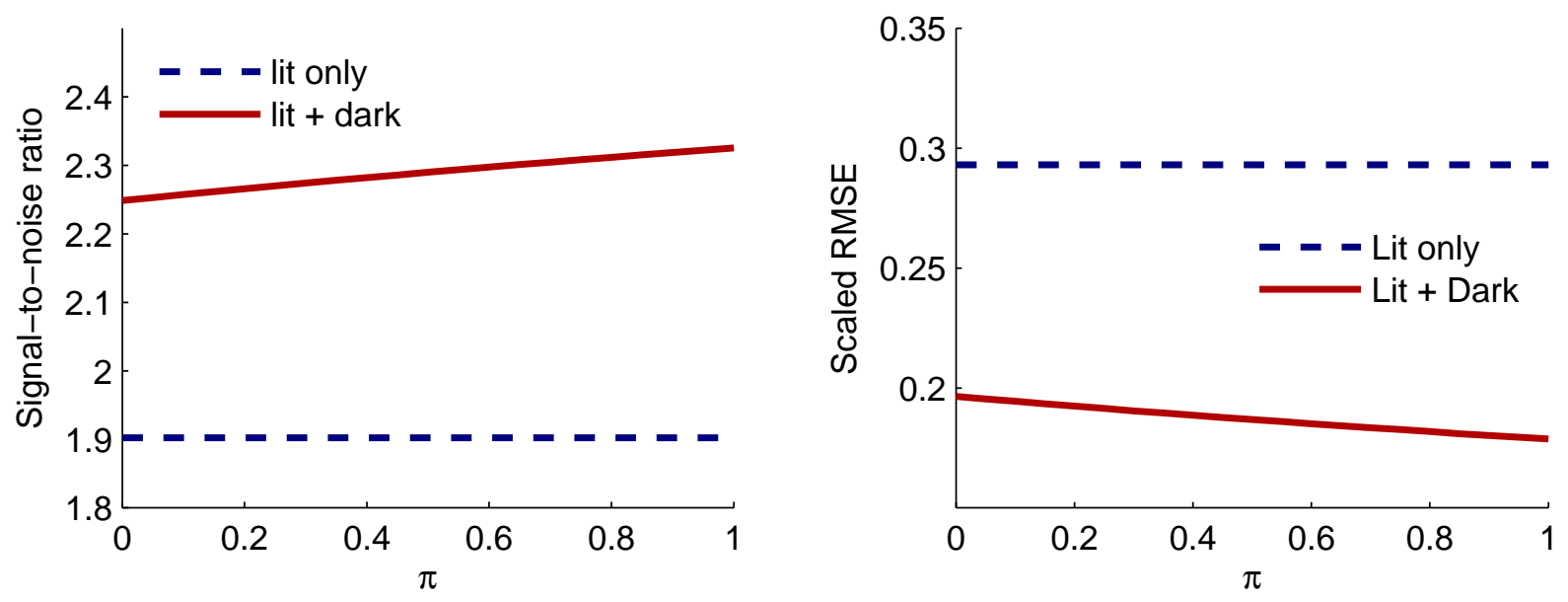

traders submit orders to the exchange in period 1 , so information horizon has no effect on the equilibrium outcome. With a dark pool, Case 2 of Proposition 6 is obtained in equilibrium. Figure 8 demonstrates that the signal-to-noise ratio is higher if the dark pool is present. This improvement, however, is smaller if the information horizon is 
longer (i.e., if $\pi$ is smaller). Similarly, the scaled RMSE is lower if a dark pool is added; conditional on having a dark pool, the RMSE is larger if the information horizon is longer (i.e., if $\pi$ is smaller).

A final observation is that information horizon relates to the frequency at which the dark pool crosses orders. Although crossing frequency is not explicitly modeled in this paper, intuition suggests that for a given information horizon (e.g., a fixed $\pi$ ), a dark pool that crosses orders less frequently poses a higher execution risk for informed traders. That is, the lower the crossing frequency, the more effective we expect the dark pool to be in improving price discovery. This result is shown in an earlier version of the paper (using a slightly different model) and is available upon request.

\section{Empirical Implications}

In this section I discuss the empirical implications of my model, both in light of recent empirical evidence and in relation to the current policy debate on the impacts of dark pools on price discovery and liquidity. In these predictions, the degree of adverse selection (or volatility $\sigma$ ), the addition of a dark pool, or the horizon of information serve as the exogenous variation and thus "cause" the effect being predicted. Whereas such causality naturally arises from an equilibrium model, identifying it in the data is more difficult. To be conservative, in the discussion below I adopt a "correlation" interpretation of recent empirical evidence. This conservative interpretation is likely to understate the results of those empirical studies and, whenever the empirical evidence is consistent with my model, understate my results as well. For a comparison of predictions from different theories of dark pools, including my model and those discussed in the introduction, see Ready (2012).

Prediction 1. All else equal, if adverse selection (or volatility) is low, then a higher adverse selection (or volatility) causes a higher dark pool market share. If adverse selection (or volatility) is high, then a higher adverse selection (or volatility) can cause a lower dark pool market share.

Prediction 1 follows from Proposition 2 (and Figure 4), where adverse selection (or volatility) is defined as the parameter $\sigma$. In my model, the first half of Prediction 1 applies when no informed traders use the dark pool, and the second half of Prediction 1 applies when at least some informed traders use the dark pool.

A robust finding in existing empirical papers is that dark pool market share is lower when volatility is higher. For example, in a sample of two block-crossing dark pools 
(Liquidnet and Posit), Ready (2012) finds that institutions are less likely to route orders to dark pools for stocks that have a higher adverse selection (defined as the component of volatility not attributable to public announcements). Using data from eleven dark pools, Buti, Rindi, and Werner (2011b) find that for a given stock, dark pool market share is higher on days with lower intraday volatility and lower absolute returns. Ye (2010) find that his sample of eight dark pools have higher market shares for stocks with lower volatility. To the extent that dark pools are used at least partially by informed traders in practice, these empirical results are consistent with the second half of Prediction 1.

Prediction 2. All else equal, a higher adverse selection (or volatility) causes more informed orders in the dark pool.

Prediction 2 also follows from Proposition 2 (i.e., $\beta \mu_{I}$ is increasing in $\sigma$ ) and is consistent with recent evidence documented by Nimalendran and Ray (2013) using data from an anonymous dark pool. They infer the trading direction of each dark-pool transaction by comparing the execution price with the prevailing market midpoint. A trading strategy that follows the directions of aggressive dark-pool orders is profitable when spreads are wide but unprofitable when spreads are narrow. To the extent that exchange spreads are proxy measures for adverse selection, Prediction 2 is consistent with their results.

Prediction 3. All else equal, adding a dark pool alongside an exchange can contemporaneously cause a higher order informativeness, wider spreads, and higher price impacts of trades on the exchange.

Prediction 3 follows directly from Proposition 3 and Proposition 4, which provide a sufficient condition for the prediction to hold. This prediction of my model is driven by the endogenous venue choices of both informed and liquidity traders, and is opposite to that of Ye (2011), who only allows informed traders to choose a trading venue.

An explicit test of Prediction 3 is to compare market quality before and after the introduction of a dark pool. In Canadian equity market, Foley, Malinova, and Park (2013) exploit the introduction of "dark orders" on the Toronto Stock Exchange, which was implemented in phases. ${ }^{28}$ Using a difference-in-difference test, they find that the introduction of dark orders widened exchange spreads. Prediction 3 is consistent with their results.

\footnotetext{
${ }^{28}$ Dark orders are fully hidden orders on exchanges. In the sample of Foley, Malinova, and Park (2013), about twothirds of dark orders on the Toronto Stock Exchange were pegged at the market midpoint. The trading mechanism of midpoint pegged orders is similar to that of midpoint dark pools.
} 
In U.S. and European equity markets, empirical studies typically use the dark pool market share as a right-hand variable and examine its effect on price discovery, liquidity, and other measures of market quality. Prediction 3 is consistent with Degryse, de Jong, and van Kervel (2011), Jiang, McInish, and Upson (2011), Weaver (2011), ComertonForde and Putnins (2012), and Nimalendran and Ray (2013). A generic result of all these studies is that higher market shares of dark pools (or dark venues) are associated with higher spreads and price impacts on exchanges. The first four studies document this result using consolidated measures of dark trading in the United States (Jiang, McInish, and Upson 2011; Weaver 2011), the Netherlands (Degryse, de Jong, and van Kervel 2011), and Australia (Comerton-Forde and Putnins 2012). Nimalendran and Ray (2013) use transaction-level data in an anonymous dark pool and find that spreads and price impacts tend to increase after dark-pool transactions.

In addition, using data from BrokerTec, an electronic trading platform for U.S. Treasury securities, Fleming and Nguyen (2013) find that transparent orders are more informative than dark orders. (Resembling a dark pool, BrokerTec's dark trading mechanism, called "workup," matches orders at the transaction price of the last market order.) The evidence in Fleming and Nguyen (2013) is also consistent with Prediction 3.

Not all empirical studies reach the same conclusion, however. For example, Buti, Rindi, and Werner (2011b) find that higher dark pool trading activity tends to be associated with lower spreads and lower return volatilities. O'Hara and Ye (2011) also conclude that higher fragmentation of trading is associated with faster execution, lower transaction costs, and more efficient prices.

A number of reasons may contribute to this disagreement. First, the studies cited above use a wide variety of data samples, and completely correcting for endogeneity is difficult. Second, the spread in my model is entirely driven by asymmetric information regarding the asset value, whereas spreads in the data can be partly driven by the level of competition and inventory cost. For example, a higher competition among trading venues or a lower inventory cost may simultaneously increase dark pool market share and reduce exchange spreads. Third, and more subtly, a higher current adverse selection on the exchange can improve price discovery so much that it reduces future spreads (hence the word "contemporaneously" in Prediction 3). In sum, Prediction 3 can explain some of the empirical evidence, but not all of it.

Prediction 4. All else equal, a shorter horizon of private information can cause a more aggressive use of dark pools by informed traders, as well as a wider exchange spread. The shorter is the information horizon, the more effective is the dark pool in improving price discovery on the exchange. 
Prediction 4 follows from Section 3.2 and is based on numerical calculations. As discussed in Section 3.2, the first part of Prediction 4 may appear surprising, but it is perfectly consistent with the equilibrium behavior of traders. A shorter information horizon makes delay more appealing for liquidity traders, driving up exchange spread; therefore, informed traders would use the dark pools more aggressively in order to save on the spread. This prediction is opposite to that of Hendershott and Mendelson (2000), where a shorter information horizon means that all informed traders send orders to the exchange immediately, and that the exchange spread becomes narrower. This distinction can be tested empirically. To the best of my knowledge, Prediction 4 has not been tested in the data. ${ }^{29}$

\section{Concluding Remarks}

Dark pools have become an important part of equity market structure. This paper provides a model of dark-pool trading and their effects on price discovery and liquidity. I show that under natural conditions the addition of a dark pool concentrates informed traders on the exchange and improves price discovery. The reporting of dark-pool volume further improves price discovery beyond the self-selection mechanism. The results of this paper challenge the conventional wisdom that dark trading is by definition harmful for price discovery. Rather, through a self-selection mechanism, adding a dark pool can improve price discovery on the exchange.

The effect of dark pools on price discovery should be balanced by a few observations. First, improved price discovery on the exchange coincides with reduced exchange liquidity, leading to ambiguous welfare implications. Second, some specifications of the delay costs can prevent liquidity traders from freely moving between the two venues, limiting the self-selection mechanism. Third, the effect of the dark pool for price discovery can become weaker the longer is information horizon. Fourth, although the dark pool can improve price discovery on average, it can harm price discovery in some rare realizations of uninformed order imbalance.

That said, evidence from several recent empirical studies reveals that the selfselection mechanism of this paper is relevant and important in reality. The evidence includes that (1) dark-pool market shares decrease in volatility, (2) trades in dark

\footnotetext{
${ }^{29}$ One possible proxy for information horizon is the time distance between trading and some scheduled news announcement, such as earnings. In this context, a corollary of Prediction 4 would be: as a news announcement becomes more imminent, exchange spread widens, and informed participation in dark pools increases. Here, informed trading can be measured by the extent to which inferred dark-pool trading ahead of a news announcement is in the same direction as the unexpected part of news announcement.
} 
venues are less informative than those in lit venues, and (3) a higher dark pool market share is associated with a higher spread and a higher price impact on the exchange. These empirical patterns are consistent with my main result that dark pools tend to attract relatively uninformed orders and, through this self-selection mechanism, improve price discovery on the exchange. 


\section{Appendix}

\section{A List of Model Variables}

This appendix summarizes key variables used in Sections 2 and 3.2.

Variable Description

\begin{tabular}{|c|c|}
\hline \multicolumn{2}{|r|}{$\underline{\text { Variables introduced in Section } 2}$} \\
\hline$v, \sigma$ & Asset value $v$ is either $+\sigma$ or $-\sigma$, for $\sigma>0$ \\
\hline $\bar{\mu}, \mu_{I}$ & Total masses of for-profit traders and informed traders \\
\hline$F$ & Cumulative distribution function (c.d.f.) of information-acquisition cost \\
\hline$Y$ & Signed informed trading interests: $Y=\operatorname{sign}(v) \cdot \mu_{I}$ \\
\hline$Z^{+}, Z^{-}, \phi$ & Liquidity buy quantity $Z^{+}$and liquidity sell quantity $Z^{-}$have p.d.f. $\phi$ \\
\hline$\mu_{z}, \sigma_{z}^{2}$ & Total mean and variance of liquidity trading interests $Z^{+}+Z^{-}$ \\
\hline$c, \gamma, G$ & Delay cost of a liquidity trader is $c=\sigma \gamma$ per unit of asset, and $\gamma$ has c.d.f. $G$ \\
\hline$\alpha_{e}, \alpha_{d}, \alpha_{0}$ & $\begin{array}{l}\text { Fractions of liquidity traders who trade on the exchange, trade in the dark } \\
\text { pool, and defer trading, respectively }\end{array}$ \\
\hline$\beta$ & Fraction of informed traders who trade in the dark pool \\
\hline$S$ & Exchange (effective) spread; bid is $-S$ and ask is $S$ \\
\hline $\bar{r}$ & Dark pool crossing probability if no informed traders go to the dark pool \\
\hline$r^{-}, r^{+}$ & $\begin{array}{l}\text { Dark pool crossing probabilities conditional on informed traders being on the } \\
\text { same and opposite side, respectively }\end{array}$ \\
\hline $\bar{\sigma}$ & Maximum volatility for which informed traders avoid the dark pool \\
\hline$\hat{\mu}_{I}(\sigma)$ & Knife-edge mass of informed traders, defined by (B1) \\
\hline$W_{e}, W_{d}$ & Expected profits of an informed buyer on the exchange and in the dark pool \\
\hline$X_{0}(c), X_{e}, X_{d}(c)$ & $\begin{array}{l}\text { Per-unit payoff of a liquidity buyer with a delay cost of } c \text { who defers trading, } \\
\text { trades on the exchange, and trades in the dark pool, respectively }\end{array}$ \\
\hline$R_{1}$ & Period-1 log likelihood ratio of $\{v=+\sigma\}$ versus $\{v=-\sigma\}$ \\
\hline$P_{1}$ & Period-1 closing price on the exchange \\
\hline$I\left(\beta, \alpha_{e}\right)$ & Signal-to-noise ratio of period-1 exchange order flow \\
\hline$R M S E$ & Root mean squared error \\
\hline$V_{b}, V_{s}$ & Period-1 realized buy volume and sell volume on the exchange, respectively \\
\hline$V_{d}, V_{e}, V$ & Expected volumes in the dark pool, on the exchange, and both, respectively \\
\hline & d in Section 3.2 \\
\hline$S_{2}$ & nounced at the beginning of period 2 \\
\hline$\gamma_{0}, \gamma_{d}$ & The marginal types of liquidity traders. $\gamma_{0}=G^{-1}\left(\alpha_{0}\right), \gamma_{d}=G^{-1}\left(1-\alpha_{e}\right)$. \\
\hline
\end{tabular}




\section{B Proofs and Details}

\section{B.1 Proof of Lemma 1}

Recall that we have assumed that each infinitesimal trader can only execute a finite number of shares per capita. Without loss of generality, we consider an informed trader who can trade up to $M$ units of shares, where $M \in(0, \infty)$. (In the main model section, $M=1$.) Since this trader is infinitesimal, his maximum order mass - the product of order size per capita and his mass - is $M \times 0=0$. Therefore, this informed trader has no effect for the exchange price or the dark-pool execution probability. Suppose that he sends $M_{e}$ shares to the exchange and $M_{d}=M-M_{d}$ shares to the dark pool. His profit is then $M_{e} W_{e}+M_{d} W_{d}$, where $W_{e}$ and $W_{d}$ do not depend on the split $\left(M_{e}, M_{d}\right)$. Clearly, if $W_{e}>W_{d}$, the optimal strategy is to set $M_{e}=M$; if $W_{d}>W_{d}$, the optimal strategy is to set $M_{d}=M$. If, however, $W_{e}=W_{d}$, then it is still optimal for the informed trader to set either $M_{e}=M$ or $M_{d}=M$, although splitting is also optimal.

\section{B.2 Proof of Proposition 1}

I define $\hat{\mu}_{I}:[0, \infty) \rightarrow[0, \bar{\mu}]$ by

$$
\hat{\mu}_{I}(s)=\bar{\mu} F\left(\frac{(1-G(1)) \mu_{z}}{\hat{\mu}_{I}(s)+(1-G(1)) \mu_{z}} s\right) .
$$

Given the value $\sigma$ of information, $\hat{\mu}_{I}(\sigma)$ is the unique "knife-edge" mass of informed traders with the property that all informed traders and a fraction $1-G(1)$ of liquidity traders send orders to the exchange.

To prove the proposition, I show that a Case 1 equilibrium exists if

$$
\bar{r} \leq 1-\frac{\hat{\mu}_{I}(\sigma)}{\hat{\mu}_{I}(\sigma)+(1-G(1)) \mu_{z}},
$$

and that a Case 2 equilibrium exists if and only if

$$
\bar{r}>1-\frac{\hat{\mu}_{I}(\sigma)}{\hat{\mu}_{I}(\sigma)+(1-G(1)) \mu_{z}} .
$$

Then I show that the condition (B2) is equivalent to $\sigma \leq \bar{\sigma}$ for some $\bar{\sigma}$, and that the condition (B3) is equivalent to $\sigma>\bar{\sigma}$.

Clearly, $\beta<1$; otherwise, the exchange spread would be zero and informed traders would deviate to trade on the exchange. Thus, in equilibrium either $\beta=0$ or $0<\beta<1$. 
We first look for an equilibrium in which $\beta=0$. By (15), $\alpha_{0}=0$ and $\alpha_{e}=$ $1-\alpha_{d}$. The indifference condition of the marginal liquidity trader is given by (16). For notational simplicity, we write the left-hand side of $(16)$ as $-\tilde{X}_{d}\left(\alpha_{d}\right)$ and the right-hand side as $-\tilde{X}_{e}\left(\alpha_{d}\right)$. For each $\alpha_{d}, \mu_{I}$ is uniquely determined by (17). We have

$$
\begin{aligned}
& -\tilde{X}_{d}(0)=0<-\tilde{X}_{e}(0), \\
& -\tilde{X}_{d}(G(1))=1-\bar{r} \geq \frac{\hat{\mu}_{I}(\sigma)}{\hat{\mu}_{I}(\sigma)+(1-G(1)) \mu_{z}}=-\tilde{X}_{e}(G(1)),
\end{aligned}
$$

where the second inequality follows from (B2), (17), and (B1). So there exists a solution $\alpha_{d}^{*} \in(0, G(1)]$ that satisfies (16).

Now we look for an equilibrium in which $\beta>0$, that is, informed traders are indifferent between the exchange and the dark pool. What remains to be shown is that the incentive-compatibility conditions (18)-(20) have a solution. For simplicity, we write the left-hand side of $(18)$ as $\tilde{W}_{d}(\beta)$ and the right-hand side of $(18)$ as $\tilde{W}_{e}(\beta)$. For each $\beta \geq 0, \mu_{I}$ is unique determined by (20) and is increasing in $\beta$. Under condition (B3) and for each $\alpha_{d}>0$,

$$
\begin{aligned}
& \tilde{W}_{d}(0)=\bar{r}>1-\frac{\hat{\mu}_{I}(\sigma)}{\hat{\mu}_{I}(\sigma)+(1-G(1)) \mu_{z}}=\tilde{W}_{e}(0), \\
& \tilde{W}_{d}(1)=r^{-}<1=\tilde{W}_{e}(1),
\end{aligned}
$$

where the first inequality follows from (B2), (17), and (B1). So there exists a solution $\beta^{*} \in(0,1)$ to (18), as a function of $\alpha_{d}$. Because $\mu_{I}$ increases in $\beta$, we see that $\tilde{W}_{d}^{\prime}(\beta)<0$ and $\tilde{W}_{e}^{\prime}(\beta)>0$, holding $\alpha_{d}$ fixed. Thus, the solution $\beta^{*}$ to (18) is unique for each $\alpha_{d}$.

Moreover, (18) implies that in equilibrium $r^{-}$is bounded away from 0. So there exists some $r_{0}>0$ such that $r^{-}>r_{0}$. So for sufficiently small $\alpha_{d}>0$,

$$
G(1)-G\left(\frac{r^{+}-r^{-}}{r^{+}+r^{-}}\right)>G(1)-G\left(\frac{1-r_{0}}{1+r_{0}}\right)>\alpha_{d} .
$$

So there exists a solution $\alpha_{d}^{*} \in(0, G(1)]$ to (19). The equilibria characterized by (18)(20) thus exist. To show that (B3) is necessary for the existence of equilibria in which $\beta>0$, suppose for contradiction that (B3) does not hold. Then, for all $\alpha_{d}$ and $\beta>0$, $\tilde{W}_{e}(\beta)>\tilde{W}_{e}(0) \geq \tilde{W}_{d}(0)>\tilde{W}_{d}(\beta)$, which implies that all informed traders wish to deviate to the exchange, contradicting $\beta>0$.

Finally, by (B1), increasing the value $\sigma$ of information raises the knife-edge mass $\hat{\mu}(\sigma)$ of informed traders, which in turn tightens the condition (B2) under which in- 
formed traders avoid the dark pool. Thus, there exists some unique volatility threshold $\bar{\sigma}$ at which (B2) holds with an equality. That is, the equilibrium in Case 1 exists if $\sigma \leq \bar{\sigma}$, and the equilibrium in Case 2 exists if $\sigma>\bar{\sigma}$.

\section{B.3 Proof of Proposition 2}

Because $\beta, \alpha_{d}, \alpha_{e}, \mu, S, r^{+}$and $r^{-}$are implicitly defined by differentiable functions in each case of Proposition 1, they are continuous and differentiable in $\sigma$ in each of the two intervals $[0, \bar{\sigma}]$ and $(\bar{\sigma}, \infty)$. At the volatility threshold $\sigma=\bar{\sigma}$, differentiability refers to right-differentiability in Case 1 of Proposition 1, and left-differentiability in Case 2.

Have a dark pool and $\sigma \leq \bar{\sigma}$

For $\sigma \leq \bar{\sigma}, \beta=0$. Total differentiation of (16)-(17) with respect to $\sigma$ yields

$$
\begin{aligned}
& \underbrace{\left[\frac{d G^{-1}\left(\alpha_{d}\right)}{d \alpha_{d}}(1-\bar{r})-\frac{\partial(S / \sigma)}{\partial \alpha_{d}}\right]}_{>0} \frac{d \alpha_{d}}{d \sigma}-\underbrace{\frac{\partial(S / \sigma)}{\partial \mu_{I}} \frac{d \mu_{I}}{d \sigma}=0,}_{>0} \\
& \underbrace{\left[1-\bar{\mu} F^{\prime}(\sigma-S) \frac{\partial(\sigma-S)}{\partial \mu_{I}}\right]}_{>0} \frac{d \mu_{I}}{d \sigma}=\underbrace{\bar{\mu} F^{\prime}(\sigma-S) \frac{\partial(\sigma-S)}{\partial \alpha_{d}}}_{<0} \frac{d \alpha_{d}}{d \sigma}+\underbrace{\bar{\mu} F^{\prime}(\sigma-S)\left(1-\frac{S}{\sigma}\right)}_{>0},
\end{aligned}
$$

where the first term of (B4) is positive because of equilibrium selection. If $d \alpha_{d} / d \sigma \leq 0$ at, say, some $\sigma_{0}$, then (B5) implies that $d \mu_{I} / d \sigma>0$ at $\sigma_{0}$. But then (B4) cannot hold. Thus, $d \alpha_{d} / d \sigma>0, d \mu_{I} / d \sigma>0$, and $d(S / \sigma) / d \sigma>0$, by (16).

\section{Have a dark pool and $\sigma>\bar{\sigma}$}

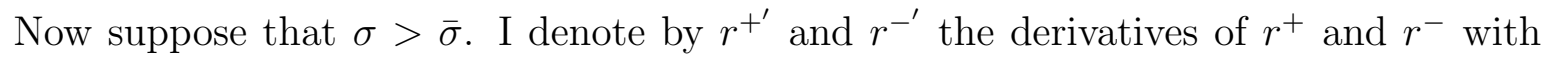
respect to $\beta \mu_{I} / \alpha_{d}$. We have $r^{+^{\prime}}>0$ and $r^{{ }^{\prime}}<0$. Total differentiation of (18)-(20) 
with respect to $\sigma$ yields

$$
\begin{aligned}
& \underbrace{\left(r^{\prime^{\prime}} \frac{1}{\alpha_{d}}-\frac{\partial(1-S / \sigma)}{\partial\left(\beta \mu_{I}\right)}\right)}_{<0} \frac{d\left(\beta \mu_{I}\right)}{d \sigma}=\underbrace{\frac{\partial(1-S / \sigma)}{\partial \mu_{I}}}_{<0} \frac{d \mu_{I}}{d \sigma}+\underbrace{r^{-^{\prime}} \frac{\beta \mu_{I}}{\alpha_{d}^{2}}}_{<0} \frac{d \alpha_{d}}{d \sigma},
\end{aligned}
$$

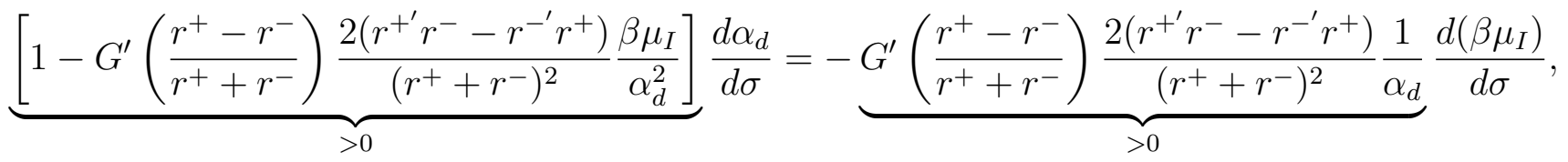

$$
\begin{aligned}
& \underbrace{\left[1-\bar{\mu} F^{\prime}(\sigma-S) \sigma \frac{\partial(1-S / \sigma)}{\partial \mu_{I}}\right]}_{>0} \frac{d \mu_{I}}{d \sigma}=\underbrace{\bar{\mu} F^{\prime}(\sigma-S) \sigma \frac{\partial(1-S / \sigma)}{\partial\left(\beta \mu_{I}\right)}}_{>0} \frac{d\left(\beta \mu_{I}\right)}{d \sigma}+\underbrace{\bar{\mu} F^{\prime}(\sigma-S)\left(1-\frac{S}{\sigma}\right)}_{>0},
\end{aligned}
$$

where the first term of (B7) is positive because of equilibrium selection.

We can show that $d \alpha_{d} / d \sigma$ cannot switch signs in $[\bar{\sigma}, \infty)$. To see why, suppose otherwise, and $d \alpha_{d} / d \sigma$ switches signs at some $\sigma_{0}$. By continuity, at $\sigma_{0}, d \alpha_{d} / d \sigma=0$. But (B7) and (B6) imply that $d\left(\beta \mu_{I}\right) / d \sigma=0=d \mu_{I} / d \sigma$ at $\sigma_{0}$ as well, which contradicts (B8). Thus, $d \alpha_{d} / d \sigma$ cannot switch signs in $[\bar{\sigma}, \infty)$; nor can it be zero.

At $\sigma=\bar{\sigma}, \beta=0$ and $d \beta / d \sigma \geq 0$. Then, by (B7),

$$
\left.\frac{d\left(\beta \mu_{I}\right)}{d \sigma}\right|_{\sigma=\bar{\sigma}}=\left.\mu_{I} \frac{d \beta}{d \sigma}\right|_{\sigma=\bar{\sigma}} \geq\left. 0 \Longrightarrow \frac{d \alpha_{d}}{d \sigma}\right|_{\sigma=\bar{\sigma}} \leq 0
$$

Because $d \alpha_{d} / d \sigma$ cannot be zero, it must be strictly negative for all $\sigma \in[\bar{\sigma}, \infty)$. By (B7)-(B8), for all $\sigma \in[\bar{\sigma}, \infty), \beta \mu_{I}$ and $\mu_{I}$ are both strictly increasing in $\sigma$. Then, (18) implies that

$$
\frac{d(S / \sigma)}{d \sigma}=-\frac{d r^{-}}{d \sigma}=-r^{-^{\prime}} \frac{d}{d \sigma}\left(\frac{\beta \mu_{I}}{\alpha_{d}}\right)>0 .
$$

The spread itself, $S=\sigma \cdot(S / \sigma)$, obviously increases in $\sigma$ as well. Finally,

$$
\frac{d r^{+}}{d \sigma}=r^{+^{\prime}} \frac{d}{d \sigma}\left(\frac{\beta \mu_{I}}{\alpha_{d}}\right)>0, \quad \frac{d r^{-}}{d \sigma}=r^{-^{\prime}} \frac{d}{d \sigma}\left(\frac{\beta \mu_{I}}{\alpha_{d}}\right)<0
$$

\section{No dark pool}

The comparative statics for Corollary 1 are similar to that for the first case of Proposition 1 and are omitted. 


\section{B.4 Proof of Proposition 3}

\section{Have a dark pool and $\sigma \leq \bar{\sigma}$}

For $\sigma \leq \bar{\sigma}$, adding a dark pool is equivalent to increasing $\bar{r}$. Total differentiation of (16)-(17) with respect to $\bar{r}$ yields

$$
\begin{aligned}
& \underbrace{\left[(1-\bar{r}) \frac{\partial G^{-1}\left(\alpha_{d}\right)}{\partial \alpha_{d}}-\frac{\partial(S / \sigma)}{\partial \alpha_{d}}\right]}_{>0} \frac{d \alpha_{d}}{d \bar{r}}=G^{-1}\left(\alpha_{d}\right)+\underbrace{\frac{\partial(S / \sigma)}{\partial \mu_{I}}}_{>0} \frac{d \mu_{I}}{d \bar{r}} \\
& \underbrace{\left[1-\bar{\mu} F^{\prime}(\sigma-S) \frac{\partial}{\partial \mu_{I}}(\sigma-S)\right]}_{>0} \frac{d \mu_{I}}{d \bar{r}}=\underbrace{\bar{\mu} F^{\prime}(\sigma-S) \frac{\partial(\sigma-S)}{\partial \alpha_{d}}}_{<0} \frac{d \alpha_{d}}{d \bar{r}},
\end{aligned}
$$

where the first term on the left-hand side of (B9) is positive because of the equilibrium selection. If $d \alpha_{d} / d \bar{r} \leq 0$ at any $\sigma_{0}$, then (B10) implies that $d \mu_{I} / d \bar{r} \geq 0$ at $\sigma_{0}$. But that contradicts (B9). Thus, $d \alpha_{d} / d \bar{r}>0$ and $d \mu_{I} / d \bar{r}<0$. Adding a dark pool, which is equivalent to an increase in $\bar{r}$, raises $\alpha_{d}$ and reduces $\alpha_{e}=1-\alpha_{d}$. The total participation rate of liquidity traders in either the dark pool or the exchange is $\alpha_{d}+\alpha_{e}=1$, higher than a market without a dark pool. Moreover, by (17), a lower $\mu_{I}$ implies a wider spread $S$ on the exchange.

\section{Have a dark pool and $\sigma>\bar{\sigma}$}

Now suppose that $\sigma>\bar{\sigma}$. In a market with a dark pool, $\alpha_{e}=1-G(1)$, a constant. Substituting it into (21) and we have

$$
\frac{\mu_{I}}{\mu_{I}+(1-G(1)) \mu_{z}}<1
$$

So the equilibrium $\alpha_{e}$ without a dark pool resides in the interval $(1-G(1), 1)$. That is, adding a dark pool reduces $\alpha_{e}$.

Moreover, adding a dark pool increases the exchange spread if and only if $\alpha_{e}$ in the equilibrium of Corollary 1 is larger than $(1-G(1)) /(1-\beta)$, where $\beta>0$ is determined in Proposition 1. By the equilibrium selection rule and by (18),

$$
\alpha_{e}>\frac{1-G(1)}{1-\beta} \Longleftrightarrow G^{-1}\left(1-\frac{1-G(1)}{1-\beta}\right)>\frac{\mu_{I}}{\mu_{I}+\mu_{z}(1-G(1)) /(1-\beta)}=1-r^{-},
$$


where the $\mu_{I}$ is given by

$$
\mu_{I}=\bar{\mu} F\left(\frac{(1-G(1)) \mu_{z}}{(1-\beta) \mu_{I}+(1-G(1)) \mu_{z}}\right)
$$

We rearrange (B11) and obtain

$$
\beta<\frac{G(1)-G\left(1-r^{-}\right)}{1-G\left(1-r^{-}\right)} .
$$

On the other hand, because the left-hand side of (18) is decreasing in $\beta$ and the righthand side is increasing in $\beta$, the above condition is equivalent to (23).

As $F(c) \rightarrow 1$ for all $c>0$, (20) implies that $\mu_{I} \rightarrow \bar{\mu}$, a constant. Holding $\mu_{I}=\bar{\mu}$ fixed, we now show that if $G^{\prime \prime}\left(1-r^{-}\right) \leq 0$, then $(23)$ holds for all $r^{-} \in[0, \bar{r}]$. At $r^{-}=\bar{r}$, we have $\sigma=\bar{\sigma}$ and (23) holds by the definition of $\bar{\sigma}$. At $r^{-}=0$, (23) also holds trivially. Take the first and second derivatives of the right-hand side of (23) with respect to $r^{-}$and we obtain

$$
\begin{aligned}
\frac{d[r h s(23)]}{d r^{-}} & =\frac{\bar{\mu} \mu_{z} G^{\prime}\left(1-r^{-}\right)}{\left[\bar{\mu}+\left(1-G\left(1-r^{-}\right)\right) \mu_{z}\right]^{2}}>0, \\
\frac{d^{2}[r h s(23)]}{d\left(r^{-}\right)^{2}} & =\bar{\mu} \mu_{z} \frac{G^{\prime \prime}\left(1-r^{-}\right)\left[\bar{\mu}+\left(1-G\left(1-r^{-}\right)\right) \mu_{z}\right]-2 \mu_{z}\left[G^{\prime}\left(1-r^{-}\right)\right]^{2}}{\left[\bar{\mu}+\left(1-G\left(1-r^{-}\right)\right) \mu_{z}\right]^{3}}<0 .
\end{aligned}
$$

Thus, the right-hand side of (23) is concave and (23) holds for all $r^{-} \in[0, \bar{r}]$.

\section{B.5 Proof of Proposition 4}

We first show that, under the normal approximation, a higher signal-to-noise ratio $I\left(\beta, \alpha_{e}\right)$ corresponds to a lower scaled RMSE $\sqrt{\mathbb{E}\left[4\left(e^{R_{1}}+1\right)^{-2} \mid v=\sigma\right]}$. We denote by $Y$ the standard normal random variable with mean zero and variance one. Conditional on $v=\sigma, R_{1}$ has the same distribution as $2 I^{2}+2 I Y$. For simplicity, in the calculation 
below I write $I\left(\beta, \alpha_{e}\right)$ as $I$ and write $R_{1}$ as $R$. Direct calculation yields:

$$
\begin{aligned}
& \frac{d}{d I} \mathbb{E}\left[\frac{1}{(\exp (R)+1)^{2}}\right]=\mathbb{E}\left[-2\left(\exp \left(2 I^{2}+2 I Y\right)+1\right)^{-3} \cdot \frac{d}{d I}\left\{\exp \left(2 I^{2}+2 I Y\right)+1\right\}\right] \\
= & \mathbb{E}\left[\frac{-2 \exp \left(2 I^{2}+2 I Y\right) \cdot(4 I+2 Y)}{\left(\exp \left(2 I^{2}+2 I Y\right)+1\right)^{3}}\right]=\mathbb{E}\left[\frac{-2 \exp \left(2 I^{2}+2 I Y\right) \cdot\left(2 I+\frac{1}{I}\left(2 I^{2}+2 I Y\right)\right)}{\left(\exp \left(2 I^{2}+2 I Y\right)+1\right)^{3}}\right] \\
= & \mathbb{E}\left[\frac{\left.\left(-4 I-\frac{2}{I} R\right) \exp (R)\right]}{(\exp (R)+1)^{3}}\right]=\int_{\mathbb{R}} \frac{\left(-4 I-\frac{2}{I} R\right) \exp (R)}{(\exp (R)+1)^{3}}\left(\frac{1}{\sqrt{2 \pi 4 I^{2}}} \exp \left(-\frac{\left(R-2 I^{2}\right)}{4 I^{2}}\right)\right) d R \\
= & \frac{\exp \left(-I^{2}\right)}{\sqrt{2 \pi 4 I^{2}}} \int_{\mathbb{R}} \frac{\left(-4 I-\frac{2}{I} R\right) \exp \left(2 R-\frac{R^{2}}{4 I^{2}}\right)}{(\exp (R)+1)^{3}} d R \\
< & \frac{\exp \left(-I^{2}\right)}{\sqrt{2 \pi 4 I^{2}}} \int_{\mathbb{R}} \frac{\left(-\frac{2}{I} R\right) \exp \left(2 R-\frac{R^{2}}{4 I^{2}}\right)}{(\exp (R)+1)^{3}} d R \\
= & -\frac{2}{I} \frac{\exp \left(-I^{2}\right)}{\sqrt{2 \pi 4 I^{2}}}\left[\int_{-\infty}^{0} \frac{R \exp \left(2 R-\frac{R^{2}}{4 I^{2}}\right)}{(\exp (R)+1)^{3}} d R+\int_{0}^{\infty} \frac{R \exp \left(2 R-\frac{R^{2}}{4 I^{2}}\right)}{(\exp (R)+1)^{3}} d R\right] \\
= & -\frac{2}{I} \frac{\exp \left(-I^{2}\right)}{\sqrt{2 \pi I^{2}}}\left[\int_{0}^{\infty} \frac{-R \exp \left(-2 R-\frac{R^{2}}{4 I^{2}}\right)}{(\exp (-R)+1)^{3}} d R+\int_{0}^{\infty} \frac{R \exp \left(2 R-\frac{R^{2}}{4 I^{2}}\right)}{(\exp (R)+1)^{3}} d R\right] \\
= & -\frac{2}{I} \frac{\exp \left(-I^{2}\right)}{\sqrt{2 \pi 4 I^{2}}} \int_{0}^{\infty} \underbrace{\left.\frac{\exp (2 R)}{(\exp (R)+1)^{3}}-\frac{\exp (-2 R)}{(\exp (-R)+1)^{3}}\right] R \exp \left(-\frac{R^{2}}{4 I^{2}}\right) d R}_{>0}]
\end{aligned}
$$

$<0$.

Thus, if adding the dark pool increases $I$, it also decreases the scaled RMSE.

Under the normal approximation, the discussion proceeding Proposition 4 establishes that $I\left(\beta, \alpha_{e}\right)$ is increasing in $S / \sigma$ (since both are increasing in $(1-\beta) \mu_{I} / \alpha_{e}$ ). The effect of adding a dark pool for signal-to-noise ratio follows directly from Proposition 3. The effect of adding a dark pool for scaled RMSE follows from the above calculation.

\section{B.6 Proof of Proposition 5}

The proof strategy is to show that, conditional on the exchange trading volumes $V_{b}$ and $V_{s}$, the dark pool trading volume cannot be equal between the two events $\{v=+\sigma\}$ and $\{v=-\sigma\}$, unless $\sigma$ and $\left(V_{b}, V_{s}\right)$ are in some null sets (i.e. sets with measure zero).

Conditional on $v=\sigma$, the market maker infers that $V_{b}=\alpha_{e} Z^{+}+(1-\beta) \mu_{I}$ and $V_{s}=\alpha_{e} Z^{-}$, from which the market maker calculates $Z^{+}$and $Z^{-}$. The single-counted 
dark pool trading volume, $\min \left(\alpha_{d} Z^{+}+\beta \mu_{I}, \alpha_{d} Z^{-}\right)$, reduces to

$$
\tilde{V}_{d}(v=+\sigma)=\frac{1}{\alpha_{e}} \min \left(\alpha_{d} V_{b}+\mu_{I}\left(\alpha_{e} \beta-\alpha_{d}(1-\beta)\right), \alpha_{d} V_{s}\right) .
$$

Conversely, conditional on $v=-\sigma$, a similar calculation implies that the single-counted dark pool trading volume is

$$
\tilde{V}_{d}(v=-\sigma)=\frac{1}{\alpha_{e}} \min \left(\alpha_{d} V_{b}, \alpha_{d} V_{s}+\mu_{I}\left(\alpha_{e} \beta-\alpha_{d}(1-\beta)\right)\right) .
$$

For the two possible dark pool volumes $\tilde{V}_{d}(v=+\sigma)$ and $\tilde{V}_{d}(v=-\sigma)$ to be equal, there are two cases: (i) $V_{b}=V_{s}$, or (ii) $\mu_{I}\left(\alpha_{e} \beta-\alpha_{d}(1-\beta)\right)=0$. The first case implies that $Z \equiv Z^{+}-Z^{-}= \pm \frac{(1-\beta) \mu_{I}}{\alpha_{e}}$, which, ex ante, happens with probability zero because the distribution of $Z$ has no point mass. For the second case, if $0<\sigma \leq \bar{\sigma}$, then $\beta=0$ and $\mu_{I}\left(\alpha_{e} \beta-\alpha_{d}(1-\beta)\right)<0$; if $\sigma>\bar{\sigma}$, then Proposition 2 implies that $\alpha_{d} / \alpha_{e}$ is decreasing in $\sigma$, which means that the equation $\alpha_{d} / \alpha_{e}=\beta /(1-\beta)$, implicit in $\sigma$, can only have a finite number of roots. Thus, $\tilde{V}_{d}(v=+\sigma)=\tilde{V}_{d}(v=-\sigma)$ only if $\sigma$ and $\left(Z^{+}, Z^{-}\right)$are in some null sets. Outside those null sets, $\tilde{V}_{d}(v=+\sigma) \neq \tilde{V}_{d}(v=-\sigma)$, and the market maker can perfectly infer $v$. This completes the proof.

\section{B.7 Proof of Proposition 6}

There are three possible cases. If $\beta=0$, then the dark pool has no adverse selection. Liquidity traders who have sufficiently small delay-cost type $\gamma$ will use the dark pool, implying $\alpha_{d}>0$ and $\alpha_{0}=0$. If $\beta>0$, then $\alpha_{d}>0$; otherwise, informed traders would not use the dark pool as they are on the same side. There are two cases for $\beta>0$, depending on $\gamma_{0}=0$ or $\gamma_{0}>0$. As before and without the loss of generality, the proof is written for informed buyers.

Case 1. $\beta=0, \alpha_{d}>0, \alpha_{0}=0$. The marginal liquidity trader, who has type $\gamma_{d}$, must be indifferent between the dark pool and the exchange. This indifference condition is given by (48). The marginal for-profit trader who is indifference between buying information or not is characterized by (49). To be consistent with the initial conjecture of $\beta=0$, (50) says that informed traders' profit on the exchange, $\sigma-S=\frac{\alpha_{e} \mu_{z}}{\mu_{I}+\alpha_{e} \mu_{z}} \sigma$, must be higher than their profit in the dark pool, $r^{-} \sigma+\left(1-r^{-}\right)(1-\pi)\left(\sigma-\mathbb{E}\left[P_{1} \mid v=\sigma\right]\right)$. Note that given $\beta=0, S_{2}=0$.

Case 2. $\beta>0, \alpha_{d}>0, \alpha_{0}>0$. In this case, there are three indifference conditions and one equation for the marginal for-profit trader. (51) is the indifference condition of a marginal informed buyer who is indifferent between the exchange and the dark pool. 
(53) is the indifference condition of a marginal liquidity trader (with type $\gamma_{d}$ ) who is indifferent between the exchange and the dark pool. (54) is the indifference condition of a marginal liquidity trader (with type $\gamma_{0}$ ) who is indifferent between the dark pool and delaying trade. In all three equations, note that a trading fee $S_{2}$ is charged if $v$ is not announced at the beginning of period 2, which happens with probability $1-\pi$. Finally, (52) is the indifference condition of the marginal for-profit trader who is indifferent between buying information or not.

Case 3. $\beta>0, \alpha_{d}>0, \alpha_{0}=0$. Under long-lived information, the expected period-2 trading cost is no longer zero, but $(1-\pi) S_{2}$. If $(1-\pi) S_{2}>\frac{r^{+}-r^{-}}{r^{+}+r^{-}} \sigma$, then a liquidity trader with type 0 (no delay cost) would prefer the dark pool to delaying trade. This incentive condition is exactly (55). As before, the indifference conditions of the marginal informed trader, the marginal for-profit trader, and the marginal liquidity trader are given by (51), (52), and (53), respectively.

\section{B.8 Equilibrium for long-lived information and exchange only}

In this appendix we characterize the equilibrium when information is potentially longlived and when there is only an exchange. Without the dark pool, $\alpha_{d}=0$ by construction. I denote by $\beta_{0}$ the fraction of informed traders who delay trading in period 1 ; the remaining fraction $1-\beta_{0}$ of informed traders send orders to the exchange immediately. Note that without a dark pool and under long-term information, it might be possible for informed traders to delay trading. The equilibrium characterization is very similar to that of Proposition 6, so the proof is omitted.

Corollary 2. In a market with only an exchange but no dark pool, there are potentially three cases of equilibrium.

(1) An equilibrium that has $\beta_{0}=0$ and $\alpha_{0}>0$ is characterized by the solution $\left(\alpha_{e}^{*}, \mu_{I}^{*}\right)$ to the equations

$$
\begin{aligned}
\frac{\mu_{I}}{\mu_{I}+\alpha_{e} \mu_{z}} & =G^{-1}\left(1-\alpha_{e}\right) \\
\mu_{I} & =\bar{\mu} F\left(\frac{\alpha_{e} \mu_{z}}{\mu_{I}+\alpha_{e} \mu_{z}} \sigma\right),
\end{aligned}
$$

subject to the incentive-compatibility condition

$$
\frac{\alpha_{e} \mu_{z}}{\mu_{I}+\alpha_{e} \mu_{z}} \sigma>(1-\pi)\left(\sigma-\mathbb{E}\left[P_{1} \mid v=\sigma\right]\right)
$$


(2) An equilibrium that has $\beta_{0}>0$ and $\alpha_{0}>0$ is characterized by the indifference conditions $W_{d}=W_{e}$ and $X_{0}\left(\gamma_{0}\right)=X_{e}$. That is, the equilibrium is given by the solution $\left(\beta^{*}, \mu_{I}^{*}, \gamma_{0}^{*}\right)$ to the equations

$$
\begin{aligned}
\frac{\alpha_{e} \mu_{z}}{\left(1-\beta_{0}\right) \mu_{I}+\alpha_{e} \mu_{z}} \sigma & =(1-\pi)\left(\sigma-\mathbb{E}\left[P_{1} \mid v=\sigma\right]-S_{2}\right), \\
\mu_{I} & =\bar{\mu} F\left(\frac{\alpha_{e} \mu_{z}}{(1-\beta) \mu_{I}+\alpha_{e} \mu_{z}} \sigma\right) \\
\frac{\left(1-\beta_{0}\right) \mu_{I}}{\left(1-\beta_{0}\right) \mu_{I}+\alpha_{e} \mu_{z}} \sigma & =\gamma_{0} \sigma+(1-\pi) S_{2}
\end{aligned}
$$

(3) An equilibrium that has $\beta_{0}>0$ and $\alpha_{0}=0$ is characterized by solutions $\left(\beta_{0}^{*}, \mu_{I}^{*}\right)$ to equations (B17) and (B18), subject to the incentive condition

$$
\frac{\left(1-\beta_{0}\right) \mu_{I}}{\left(1-\beta_{0}\right) \mu_{I}+\mu_{z}} \sigma<(1-\pi) S_{2}
$$




\section{References}

Admati, A. R., and Pfleiderer, P. 1988. A Theory of Intraday Patterns: Volume and Price Variability. Review of Financial Studies 1: 3-40.

Bennett, J., Colon, J., Feng, J., and Litwin, J. 2010. Ten Questions Every Institution Should Ask Their Dark Pool Providers. Journal of Trading 5: 10-13.

Benveniste, L. M., Marcus, A. J., and Wilhelm, W. J. 1992. What's Special about the Specialist?. Journal of Financial Economics 32: 61-68.

Bloomfield, R. J., O'Hara, M., and Saar, G. 2012. Hidden Liquidity: Some New Light on Dark Trading. Working paper Cornell University.

Bond, P., Edmans, A., and Goldstein, I. 2012. The Real Effects of Financial Markets. Forthcoming, Annual Reviews of Financial Economics.

Boni, L., Brown, D. C., and Leach, J. C. 2012. Dark Pool Exclusivity Matters. Working paper.

Boulatov, A., and George, T. J. 2013. Securities Trading when Liquidity Providers are Informed. Review of Financial Studies 26: 2096-2137.

Brandes, Y., and Domowitz, I. 2010. Alternative Trading Systems in Europe: Trading Performance by European Venues Post-MiFID. Journal of Trading 5: 17-30.

Brunnermeier, M. K., and Pedersen, L. H. 2005. Predatory Trading. Journal of Finance 60: 1825-1863.

Brunnermeier, M. K., and Pedersen, L. H. 2009. Market Liquidity and Funding Liquidity. Review of Financial Studies 22: 2201-2238.

Buti, S., Rindi, B., Wen, Y., and Werner, I. M. 2011. Tick Size Regulation, Intermarket Competition and Sub-Penny Trading. Working paper.

Buti, S., Rindi, B., and Werner, I. M. 2011a. Dark Pool Trading Strategies. Working paper.

Buti, S., Rindi, B., and Werner, I. M. 2011b. Diving into Dark Pools. Working paper Fisher College of Business, Ohio State University.

Butler, G. 2007. Liquidity Aggregation: What Institutional Investors Need to Know. Journal of Trading 2: 108-113.

Carlin, B. I., Lobo, M. S., and Viswanathan, S. 2007. Episodic Liquidity Crisis: Cooperative and Predatory Trading. Journal of Finance 62: 2235-2274.

Carrie, C. 2008. Illuminating the New Dark Influence on Trading and U.S. Market Structure. Journal of Trading 3: 40-55. 
CFA Institute 2009. Market Microstructure: The Impact of Fragmentation under the Markets in Financial Instruments Directive. Technical report CFA Institute.

Chakravarty, S., and Holden, C. W. 1995. An Integrated Model of Market and Limit Orders. Journal of Financial Intermediation 4: 213-241.

Chordia, T., Roll, R., and Subrahmanyam, A. 2011. Recent Trends in Trading Activity and Market Quality. Journal of Financial Economics 101: 243-263.

Chowdhry, B., and Nanda, V. 1991. Multimarket Trading and Market Liquidity. Review of Financial Studies 4: 483-511.

Comerton-Forde, C., and Putnins, T. 2012. Dark trading and price discovery. Working paper.

Conrad, J., Johnson, K. M., and Wahal, S. 2003. Institutional trading and alternative trading systems. Journal of Financial Economics 70: 99-134.

CSA/IIROC 2009. Dark Pools, Dark Orders, and Other Development in Market Structure in Canada. Consulation paper 23-404 Canadian Securities Administration and Investment Industry Regulatory Organization of Canada.

Degryse, H., de Jong, F., and van Kervel, V. 2011. The Impact of Dark and Visible Fragmentation on Market Quality. Working paper.

Degryse, H., Van Achter, M., and Wuyts, G. 2009. Dynamic Order Submission Strategies with Competition between a Dealer Market and a Crossing Network. Journal of Financial Economics 91: 319-338.

Domowitz, I., Finkelshteyn, I., and Yegerman, H. 2009. Cul de Sacs and Highways, An Optical Tour of Dark Pool Trading Performance. Journal of Trading 4: 16-22.

Easley, D., Keifer, N. M., and O'Hara, M. 1996. Cream-Skimming or Profit-Sharing? The Curious Role of Purchased Order Flow. Journal of Finance 51: 811-833.

European Commission 2010. Review of the Markets in Financial Instruments Directive (MiFID). Public consultation European Commission.

Fleming, M., and Nguyen, G. 2013. Order Flow Segmentation and the Role of Dark Trading in the Price Discovery of U.S. Treasury Securities. Working paper.

Foley, S., Malinova, K., and Park, A. 2013. Dark Trading on Public Exchanges. Working paper.

Foster, D., Gervais, S., and Ramaswamy, K. 2007. The Benefits of Volume-Conditional Order-Crossing. Working paper.

Foucault, T. 1999. Order Flow Composition and Trading Costs in a Dynamic Limit Order Market. Journal of Financial Markets 2: 99-134. 
Foucault, T., Kadan, O., and Kandel, E. 2005. Limit Order Book as a Market for Liquidity. Review of Financial Studies 18: 1171-1217.

Garfinkel, J. A., and Nimalendran, M. 2003. Market Structure and Trader Anonymity: An Analysis of Insider Trading. Journal of Financial and Quantitative Analysis 38: 591-610.

Glosten, L. R., and Milgrom, P. R. 1985. Bid, Ask and Transaction Prices in a Specialist Market with Heterogeneously Informed Traders. Journal of Financial Economics 14: 71-100.

Goettler, R. L., Parlour, C. A., and Rajan, U. 2005. Equilibrium in a Dynamic Limit Order Market. Journal of Finance 60: 2149-2192.

Goettler, R. L., Parlour, C. A., and Rajan, U. 2009. Informed Traders and Limit Order Markets. Journal of Financial Economics 93: 67-87.

Grammig, J., Schiereck, D., and Theissen, E. 2001. Knowing Me, Knowing You: Trader Anonymity and Informed Trading in Parallel Markets. Journal of Financial Markets 4: $358-412$.

Guerrieri, V., and Shimer, R. 2012. Dynamic Adverse Selection: A Theory of Illiquidity, Fire Sales, and Flight to Quality. Working paper. University of Chicago.

Hendershott, T., and Jones, C. M. 2005. Island Goes Dark: Transparency, Fragmentation, and Regulation. Review of Financial Studies 18: 743-793.

Hendershott, T., and Mendelson, H. 2000. Crossing Networks and Dealer Marekts: Competition and Performance. Journal of Finance 55: 2071-2115.

Holden, C. W., and Subrahmanyam, A. 1992. Long-Lived Private Information and Imperfect Competition. Journal of Finance 47: 247-270.

International Organization of Securities Commissions 2010. Issues Raised by Dark Liquidity. Consultation report Technical Committee of the International Organization of Securities Commissions.

International Organization of Securities Commissions 2011. Principles for Dark Liquidity. Final report Technical Committee of the International Organization of Securities Commissions.

Jiang, C., McInish, T., and Upson, J. 2011. Why Fragmented Markets Have Better Market Quality: The Flight of Liquidity Order Flows to Off Exchange Venues. Working paper.

Johnson, B. 2010. Algorithmic Trading \&5 DMA: An introduction to direct access trading strategies: . 4Myeloma Press, London.

Kaniel, R., and Liu, H. 2006. So What Orders Do Informed Traders Use?. Journal of Business 79: 1867-1913. 
Kyle, A. S. 1985. Continuous Auctions and Insider Trading. Econometrica 53: 13151335 .

Madhavan, A., and Cheng, M. 1997. In Search of Liquidity: Block Traders in the Upstairs and Downstairs Markets. Review of Financial Studies 10: 175-203.

Mittal, H. 2008. Are You Playing in a Toxic Dark Pool? A Guide to Preventing Information Leakage. The Journal of Trading 3: 20-33.

Næs, R., and Odegaard, B. A. 2006. Equity trading by institutional investors: To cross or not to cross?. Journal of Financial Markets 9: 79-99.

Nimalendran, M., and Ray, S. 2013. Informational Linkages Between Dark and Lit Trading Venues. Forthcoming, Journal of Financial Markets.

O'Hara, M. 2003. Presidential Address: Liquidity and Price Discovery. Journal of Finance 58: 1335-1354.

O'Hara, M., and Ye, M. 2011. Is Market Fragmentation Harming Market Quality?. Journal of Financial Economics 100: 459-474.

Pagano, M. 1989. Trading Volume and Asset Liquidity. Quarterly Journal of Economics 104: 255-274.

Parlour, C. 1998. Price Dynamics in Limit Order Markets. Review of Financial Studies 11: 789-816.

Parlour, C. A., and Seppi, D. J. 2008. Limit Order Markets: A Survey. Chapter 3, Handbook of Financial Intermediation and Banking.

Ready, M. J. 2012. Determinants of Volume in Dark Pools. Working paper University of Wisconsin-Madison.

Rosenblatt Securities 2011. Let there be light. Market structure analysis.

Rosu, I. 2009. A Dynamic Model of the Limit Order Book. Review of Financial Studies 22: 4601-4641.

Saraiya, N., and Mittal, H. 2009. Understanding and Avoiding Adverse Selection in Dark Pools. Working paper Investment Technology Group.

Securities and Exchange Commission 2005. Regulation NMS. Federal Register 70: 37495-37644.

Securities and Exchange Commission 2009. Regulation of Non-Public Trading Interests; Proposed Rule. Federal Register 74: 61207-61238.

Securities and Exchange Commission 2010. Concept Release on Equity Market Structure; Proposed Rule. Federal Register 75: 3593-3614. 
Seppi, D. 1990. Equilibrium Block Trading and Asymmetric Information. Journal of Finance 45: 73-94.

Sofianos, G., and Xiang, J. 2011. Dark Pool Races, Part Two. Working paper. Goldman Sachs.

Sun, Y. 2006. The exact law of large numbers via Fubini extension and characterization of insurable risks. Journal of Economic Theory 126: 31-69.

Tabb Group 2011. Liquidity Matrix. Technical report.

Weaver, D. 2011. Off-Exchange Reporting and Market Quality in a Fragmented Market Structure. Working paper.

Ye, M. 2010. Non-execution and Market Share of Crossing Networks. Working paper University of Illinois.

Ye, M. 2011. A Glimpse into the Dark: Price Formation, Transaction Costs and Market Share of the Crossing Network. Working paper University of Illinois. 